\title{
A technique for resolving the spectral response of a wide-band infrared measuring instrument from measurements without the need for a spectrally tuneable radiation source
}

\author{
S. J. P. Retief \\ University of Pretoria, Hatfield, South Africa and Denel Dynamics, P.O. Box 7412, Centurion, 0046, South Africa \\ E-mail address (corresponding author): paul.retief@deneldynamics.co.za; phone +27 12671 1817; www.deneldynamics.co.za
}

\begin{abstract}
A technique is presented in which the spectral response of a wide-band infrared measuring instrument is obtained by means of calibration type measurements of a blackbody at various temperature settings and the application of a mathematical technique to these measurements. Successful application of the developed technique is enabled by Tikhonov regularization, which ensures a stable solution to the ill-posed problem of the Fredholm integral equation of the first kind describing the blackbody measurements made by the instrument. The quality of the solution strongly depends, however, on the accuracy of the measurements and the accuracy of the modelled spectrum in describing the emission from the blackbody source.

The technique is studied by means of an application to modelled results of instrument measurements, and is then applied to actual measurements made with an infrared camera. It is shown that the technique can, in the absence of specific manufacturer supplied information of the instrument and specialized equipment, be an alternative to the theoretical calculation of the instrument response spectrum or to an experimental determination of the instrument spectral response during which measurements are made of a spectrally tuneable radiation source like a blackbody and monochromator.
\end{abstract}

Keywords: Infrared measurements, spectral response, Fredholm integral of the first kind, Tikhonov regularization.

\section{INTRODUCTION}

In the field of radiometry, the infrared characteristics of an object of interest are inferred from radiation measurements done with calibrated instruments like cameras and spectrometers [1]. Such instruments, which are in common use in the military environment, usually function in one of the atmospheric windows in which attenuation by the atmospheric constituents allows a reasonable distance of propagation of the infrared radiation before total extinction. These windows, or spectral bands, are loosely defined as near-infrared (NIR, $0.75-1.4 \mu \mathrm{m}$ ), short-wave infrared (SWIR, $1.5-2.5 \mu \mathrm{m}$ ), medium-wave (or mid-wave) infrared (MWIR, $3-5 \mu \mathrm{m}$ ) and long-wave infrared (LWIR, 8-12 $\mu \mathrm{m}$ ) [2]. The optical systems associated with these instruments, typically composed of lenses, reflective elements, windows, filters and anti-reflection coatings, need to be transparent (or fully reflective) to the infrared wavelengths within one (or several) of the mentioned spectral bands in order for the radiated energy from the object of interest to reach the detector. The detector itself needs to be designed and manufactured as to be sensitive in the required wavelength band.

In practice, no optical system is fully transparent at all wavelengths [3] within a spectral band, and the sensitivity of the widely used infrared photon detectors can also be assumed to have a non-flat spectral response to incoming radiation [4]. The response of the other main type of detectors in common use, i.e. thermal detectors, is generally wavelength independent [4], but spectral selectivity is also introduced to this type of detector whenever a surface coating with selective spectral absorptivity properties is applied to the detector. The combined effects of spectral selectivity in the optical system transmission and detector response result in the instrument, as a whole, having a non-flat spectral response to any radiation measured by it.

The influence of a non-flat instrument spectral response can largely be factored out by a calibration procedure. During this procedure an infrared source, like a blackbody with known Planck distributions of radiated energy at different temperature settings, is used as the object of interest to be measured by the instrument. The output signals of the instrument can then be correlated with the known temperature or radiance values of the blackbody being measured, so that the non-flat spectral instrument response is compensated for. In such a way, the instrument signal obtained from measuring a blackbody of unknown temperature can be inferred from the calibration information. However, for objects with non-blackbody spectra, such as spectrally selective emitters like gases, or grey bodies with constant emissivities smaller than one, the inferred temperature will most probably not agree with the object's thermodynamic temperature and is called the radiometric temperature (see [5] for the definitions of several types of radiometric temperatures). Moreover, for measurements during which the infrared signature of an object must be determined over a 
distance for which the atmospheric and other environmental contributions to the observed radiation is nonnegligible, the measured radiation (and associated radiometric temperature), digress even more from the true value of that of the object of interest. In order to correctly model the radiance measured by the instrument, or to attempt to infer the source radiance value of the object of interest during data reduction by correcting for exogenous influences on the measurement, the instrument spectral response is required [6].

In general, the instrument spectral response can be determined by one of two methods:

- Theoretically by having information from the manufacturer in hand, like the detector responsivity as calculated from the spectral quantum efficiency of a photon detector [7], the spectral absorption of the surface of a thermal detector and the spectral transmission of the optical system and any filter or coating forming part of it.

- Purely experimentally with the use of a spectrally tuneable radiation source, like an infrared source and monochromator of some sort, capable of generating infrared radiance within a narrow wavelength band of which the centre frequency can be swept through the spectral wide-band (NIR, SWIR, MWIR or LWIR) in which the instrument operates, e.g. [8].

The first method typically, in practice, suffers from incomplete or unavailable information. Furthermore, the spectral information supplied by a manufacturer most likely consists only of typical values of quantum efficiency and transmission for a batch of detectors and optical systems, instead of the exact values of the specific component purchased.

The second method can be considered as the preferred method, since any manufacturing deviation from the design norm is automatically apprehended in the measurements. The problem here might be the lack of the appropriate, specialized, and usually costly, equipment. Also, due to the nature of a narrow band radiation source, the amount of radiation energy decreases as the resolution (determined by the width of the tuneable narrow wavelength band) increases, which might present instrument dependent problems related to inadequate sensitivity.

The goal of the research reported on in this article is to present a promising alternative method to the two mentioned traditional methods for determining the spectral response of an infrared measuring instrument. The main idea behind this method is to use only a blackbody, which is a standard piece of equipment in any infrared laboratory, and a very low temperature object for calibration type measurements from which the instrument spectral response can be retrieved after applying an appropriate mathematical technique.

In Section 2.1 the mathematical technique is developed by starting from the basic measurement equation for calibration type measurements. The technique is considered novel in the sense that it demonstrates that it is in principle possible to obtain the instrument spectral response of a wide-band instrument from measurements of a blackbody without the use of a monochromator. However, although the spectral response recovery is in principle possible, it does come with complications arising from the instability of the solution. Fortunately, an attempt can be made to stabilize this type of unstable solution arising from the inversion of ill-conditioned matrices; to this effect, the regularization method of Tikhonov is introduced. After introduction of this stabilization method, the technique is applied in Section 2.2 to synthetically generated measurements, i.e. measurement results with no measurement errors but with unstable solutions. The behaviour of the technique with the mentioned regularization method is then demonstrated by an investigation into its dependency on the controllable aspects, i.e. controllable parameters, used during the process of obtaining a solution, followed by a discussion of the findings in Section 2.3.

Section 3.1 presents the spectral response of a real instrument, a MWIR camera, as obtained from the two earlier mentioned traditional methods. In order to utilize the proposed technique for obtaining the camera spectral response, it is showed in Section 3.2 how a realistic model of the signal output from the instrument should be constructed before attempting to resolve its spectral responsivity. This model is considered sufficiently generic to be applicable to a wide range of infrared (IR) camera models. The measurement setup of the calibration type measurements is then also described in detail to enable any interested party to replicate these types of measurements. It is then shown that, although it is in principle possible to resolve the spectral response of an instrument by the proposed technique as illustrated in Section 2, a high degree of accuracy are required in the measurements and in the knowledge of the observed object radiance spectrum. The purely experimentally determined camera spectral response (second method described above), considered as an absolute reference, is then used to compare the solutions obtained from the proposed technique against and also to obtain an indication of the required degree of measurement accuracy for the technique to be applied successfully. 
Section 4 summarizes the research done and the findings and limitations when applying the proposed technique, with some reflections on possible future research and other applications.

\section{TECHNIQUE FOR RESOLVING THE INSTRUMENT SPECTRAL RESPONSE}

\subsection{Theory}

The measurement of an object by an ideal instrument, without any exogenous influences, can be described by the measurement equation (cf. [9])

$$
s=\int_{\lambda_{a}}^{\lambda_{b}} L_{o b j}(\lambda) \Re_{L}(\lambda) d \lambda,
$$

where $s$ (units of $[\mathrm{V}]$ or $[\mathrm{A}]$ ) is the signal obtained from the instrument as a result of the observed object having a wavelength $(\lambda)$ dependent radiance $L_{o b j}(\lambda)\left[W \cdot m^{-2} \cdot s r^{-1} \cdot \mu m^{-1}\right]$. The wavelengths $\lambda_{a}$ and $\lambda_{b}$ demarcate the spectral band in which the instrument is sensitive to radiation, and $\mathfrak{R}_{L}(\lambda)$ is the spectral radiance responsivity of the instrument, which is the ratio of the output of the instrument to that of the spectral radiance of the observed object (units of $\left[(\mathrm{V}\right.$ or $\left.\left.\mathrm{A}) /\left(W \cdot \mathrm{m}^{-2} \cdot s r^{-1} \cdot \mu \mathrm{m}^{-1}\right)\right]\right)$. The responsivity can be written as

$$
\Re_{L}(\lambda)=\Re_{L}\left(\lambda_{o}\right) r(\lambda)=C_{L 2 s} r(\lambda),
$$

where $C_{L 2 s} \equiv \Re_{L}\left(\lambda_{0}\right)$ is the spectral responsivity at the wavelength at which $\Re_{L}$ obtains its maximum value, i.e. at wavelength $\lambda_{o}$, so that $r(\lambda)=\mathfrak{R}_{L}(\lambda) / C_{L 2 s}$ forms the normalized spectral responsivity, which are often used in these type of measurement equations (see e.g. [10-12]). $C_{L 2 s}$ forms a wavelength independent conversion factor (and therefore removable from under the integration sign in Equation (1)), which both scales the calculated radiance back to the correct level and also converts it into instrument signal units ( $L$ to $s$ ), whenever the normalized spectral responsivity $r$ is used in the measurement equation as is shown later in Equation (4). The use of $r$ introduces a simplification in the notation used in the technique under development, with $r$ containing only the essence of the desired information to be retrieved- the shape of the spectral response and not so much the absolute level of this spectrum.

The object radiance, $L_{o b j}(\lambda)$, describes all contributions from all sources of radiance reaching the instrument, but in the rest of this section it will be considered to consist only of a blackbody, described by Planck's law

$$
L_{b b}(T, \lambda)=\frac{2 h c^{2}}{\lambda^{5}} \frac{1}{e^{\frac{h c}{\lambda k T}}-1},
$$

with $L_{b b}(T, \lambda)$ being the blackbody radiance, $c$ the velocity of light, $h$ the Planck constant, $k$ the Boltzmann constant and $T$ the temperature of the blackbody. The implied assumption when setting $L_{o b j}=L_{b b}$ in the measurement equation, is that the radiance observed by the instrument is only from this one source having a fixed temperature $T$ and a constant spectral emissivity of $\varepsilon=1$, and that there are no exogenous influences on this blackbody measurement from the atmosphere or any other sources, like the thermal radiation from the camera itself. This assumption will serve in assisting the demonstration of the proposed technique, but will be adapted in Section 3 when real measurements are used in the application of the technique. Eq. (1) can now be rewritten as

$$
s(T)=C_{L 2 s} \int_{\lambda_{a}}^{\lambda_{b}} L_{b b}(T, \lambda) r(\lambda) d \lambda \equiv C_{L 2 s} L_{p n}(T),
$$

describing the signal obtained from the measuring instrument when observing a blackbody with temperature $T$, for which the radiance spectrum $L_{b b}(T, \lambda)$ is theoretically known. The peak-normalized radiance $[13,14]$ for the instrument specific $r(\lambda)$, as defined in the above equation, is denoted by $L_{p n}(T)$. It must be noted that Eq. (4) is a Fredholm Integral equation of the First Kind (IFK) [15-17], which have the generic form $g(y)=$ $\int_{x_{1}}^{x_{2}} K(y, x) f(x) d x$, where the kernel $K(y, x)$ is a known function of the two variables $x$ and $y, f(x)$ is an unknown function to be solved and $g(y)$ is known for different values of $y$, usually obtained from measurements. With the kernel in Eq. (4) being Planck's law, the goal is now to obtain the unknown $r(\lambda)$, or 
$C_{L 2 s} r(\lambda)$, after which the value of $C_{L 2 s}$ can also be obtained in a straightforward manner. Note that the $L_{b b}(T, \lambda)$ kernel is not separable into factors containing only $T$ and $\lambda$ respectively, which negates the possibility of removing $T$ from under the integral sign, thereby complicating any analytical solution.

The first step is to discretize the integral in Eq. (4) by means of a quadrature formula. A closed quadrature formula for numerical integration yields

$$
L_{p n}(T) \approx \sum_{j=1}^{n} w_{j} L_{b b}\left(T, \lambda_{j}\right) r\left(\lambda_{j}\right)
$$

with $\lambda_{1}=\lambda_{a}$ and $\lambda_{n}=\lambda_{b}$, where $w_{j}$ are the weights associated with the chosen quadrature formula. For equally spaced wavelength intervals $\Delta \lambda=\lambda_{i+1}-\lambda_{i}$, the extended trapezoidal rule [16] prescribes the weights to be $w_{1}=w_{n}=\Delta \lambda / 2$ and $w_{2}=w_{3}=\cdots=w_{n-1}=\Delta \lambda$. For each measurement made of a blackbody at a specific temperature $T_{i}$, as is done during calibration of an instrument, Eq. (4) can now be written as

$$
s_{i}=C_{L 2 s} L_{p n}\left(T_{i}\right) \approx C_{L 2 s} \sum_{j=1}^{n} w_{j} L_{b b}\left(T_{i}, \lambda_{j}\right) r\left(\lambda_{j}\right)=C_{L 2 s} \sum_{j=1}^{n} L_{i, j} r_{j},
$$

where the shorthand $s_{i} \equiv s\left(T_{i}\right), L_{i, j} \equiv w_{j} L_{b b}\left(T_{i}, \lambda_{j}\right)$ and $r_{j} \equiv r\left(\lambda_{j}\right)$ is used. Each of $m$ temperature measurements can be described by Eq. (6), thereby forming a system of equations, which can be written in matrix form as

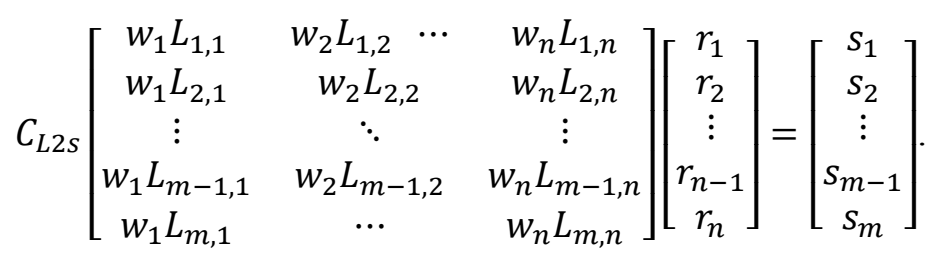

If

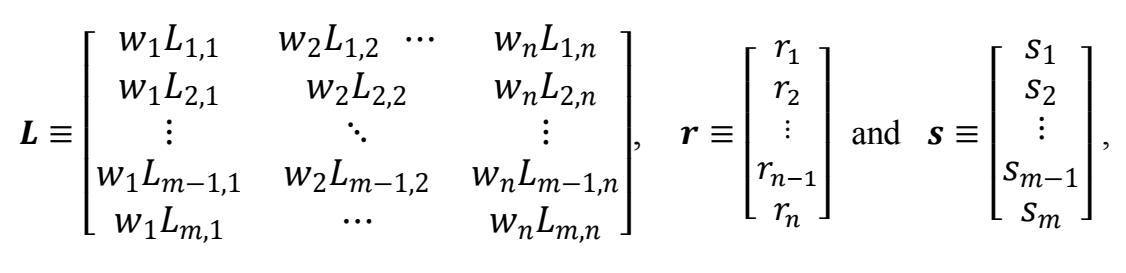

The system of calibration measurement equations, given by Eq. (7), can now be written in a more compact form as

$$
C_{L 2 s} \operatorname{Lr}=\boldsymbol{s}
$$

The columns of the radiance matrix $\boldsymbol{L}$ represent the model space $\mathbb{R}^{n}$, with its dimension $n$ being determined by the number of wavelengths over which the model (blackbody radiance) is described. The rows of $\boldsymbol{L}$ represent the data space $\mathbb{R}^{m}$, with its dimension $m$ being determined by the number of measurements available. If $m=n$, the system is square and an exact solution might exist. If $m<n$, the system is underdetermined with infinitely many or no solutions, which is not useful in obtaining a unique solution of $\boldsymbol{r}$. If $m \geq n$, the system is square or overdetermined and a least-squares (LS) solution can be obtained. In the context of this work, Eq. (7) is considered to represent either a square or an overdetermined system of equations with the number of wavelength nodes $n$, at which the blackbody emission (model) values are calculated, being the same or less than the number of blackbody temperatures $m$. It, however, makes sense to use the maximum allowable value of the modelling parameter $n$ without the system being underdetermined, so that $n=m$ is mostly used in the remainder of the work, except where the effect of $n<m$ will be 
illustrated later. The aim with a LS solution is to minimize the Euclidian residual vector norm, i.e. it is attempted to obtain

$$
\min \left\|C_{L 2 s} \boldsymbol{L r}-\boldsymbol{s}\right\|
$$

The solution for $\boldsymbol{r}$ in Eq. (10) is obtained by multiplying both sides of Eq. (9) from the left by the transpose of $\boldsymbol{L}$, i.e. $\boldsymbol{L}^{\mathrm{T}}$, and then multiplying both sides from the left by $\frac{1}{C_{L 2 s}}\left(\boldsymbol{L}^{T} \boldsymbol{L}\right)^{-1}$, giving the set of normal equations

$$
r=\frac{1}{C_{L 2 S}}\left(\boldsymbol{L}^{T} L\right)^{-\mathbf{1}} \boldsymbol{L}^{T} S=\frac{1}{C_{L 2 S}} \tilde{L}^{-1} L^{T} S
$$

with $\tilde{\boldsymbol{L}} \equiv \boldsymbol{L}^{T} \boldsymbol{L}$

Eq. (11) has a unique solution whenever matrix $\tilde{\boldsymbol{L}}$ is invertible (and $\boldsymbol{s} \neq \mathbf{0}$ ) [16]. Unfortunately, IFKs are often extremely ill-conditioned [16], with very unpleasant properties inherent to them [15], and are therefore not easily invertible. It is in fact stated by Wing [15], that "A solution to ... the IFK ... can seldom be obtained in closed form. Frequently, in practical situations, a reliable approximation to the solution cannot even be found ... It is usually necessary to settle for less information than we would like". The author then states that he prefers to rather speak of resolving, than solving, IFK problems, a preference which will be maintained in this article as far as possible synonymously with the term recovered. Wing also states that it is clear from the unboundedness of the inverse kernel and the fact that small changes in the measured data $(\boldsymbol{s}$ in this context) may produce "wild" behaviour in the solution $(\boldsymbol{r})$, that a means must often be found to "tame" this solution function; "Researchers should be quite aware that they have a great deal of control over and responsibility for this taming". The making of subjective judgements and the use of insight, intuition and educated guesses are then stated as necessities in solving IFK problems. Inverse blackbody radiation problems, similar to the one addressed here but for which a different parameter than emissivity is sought, are no different $[18,19]$. It is the intention to show in this article what is required to tame the spectral response solution function, and to show the degree of success of what can be achieved with error free synthetic data as well as data containing uncertainties from a MWIR camera.

In order to easily diagnose the condition of $\tilde{\boldsymbol{L}}$ (i.e. how invertible it is), the singular value decomposition (SVD) technique is firstly be used to factor $\boldsymbol{L}$ (being an $m \times n$ matrix) into an $m \times m$ orthogonal matrix $\boldsymbol{U}$, an $n \times n$ diagonal matrix $\boldsymbol{D}$ having singular values $d_{i}$ on the diagonal and the transpose of an $n \times n$ orthogonal matrix $\boldsymbol{V}[16]$, i.e.

$$
\boldsymbol{L}=\boldsymbol{U} \boldsymbol{D} \boldsymbol{V}^{T}
$$

The SVD of $\boldsymbol{L}$ then allows easy calculation of the condition number, $C$, of $\tilde{\boldsymbol{L}}$ since

$$
C(\tilde{\boldsymbol{L}})=\frac{d_{\max }^{2}}{d_{\min }^{2}}
$$

where $d_{\max }$ and $d_{\min }$ are the largest and smallest singular values amongst $d_{i}$ respectively. This condition number assists in determining the behaviour of $\tilde{\boldsymbol{L}}$ upon inversion - if $C=\infty$, the matrix is singular; if $C \gg 1$ (not infinite) the matrix is ill-conditioned; if $C$ is closer to one, the matrix is well-conditioned $[15,16,20]$.

The approach towards the stable inversion of $\tilde{\boldsymbol{L}}$ in this work is by means of the regularization method of Tikhonov $[15,17,19]$. In this method, the minimization of the residual vector norm in Eq. (10) is replaced by the penalized LS problem

$$
\min \left\{\left\|C_{L 2 s} \boldsymbol{L r}-\boldsymbol{s}\right\|^{2}+\alpha\left\|C_{L 2 s} \boldsymbol{r}\right\|^{2}\right\},
$$

i.e. solutions of large norm $\boldsymbol{r}$ is now penalized, with the scalar $\alpha>0$ being called the regularization parameter. The first term, when small, guarantees that $\boldsymbol{r}$ is 'nearly' a LS solution, while the second term tends to damp out instabilities in $\boldsymbol{r}$ [19]. The balance between the two terms, i.e. the normal LS solution of $\boldsymbol{r}$ and the damping of instabilities in $\boldsymbol{r}$, is determined by the value of $\alpha$ (some literature uses $\alpha^{2}$ ). This regularized version of Eq. (10) now represents a well-posed problem from which a well behaved solution should be obtainable. The LS solution for Eq. (14) is

$$
\boldsymbol{r}_{\alpha}=\frac{1}{C_{L 2 s}}(\tilde{\boldsymbol{L}}+\alpha \boldsymbol{I})^{-1} \boldsymbol{L}^{T} \boldsymbol{s},
$$


where $\boldsymbol{I}$ is an identity matrix with the same dimensions as $\tilde{\boldsymbol{L}}$. The solution is unique for a specific value of $\alpha$ and is called the Tikhonov approximation to $\boldsymbol{L}^{\dagger} \boldsymbol{s}$, the minimum norm solution of the normal equations, where $\boldsymbol{L}^{\dagger}$ is the Moore-Penrose generalized inverse of $\boldsymbol{L}$ [19]. It can be seen that Eq. (15) is identical to Eq. (11), except for $\tilde{\boldsymbol{L}}$ which is now replaced by $\tilde{\boldsymbol{L}}+\alpha \boldsymbol{I}$, i.e. the scalar value $\alpha$ was added to all elements on the diagonal of $\tilde{\boldsymbol{L}}$.

Substitution of Eq. (12) into Eq. (15) results in the SVD implementation of the Tikhonov regularization [17]

$$
\boldsymbol{r}_{\alpha}=\frac{1}{C_{L 2 S}} \boldsymbol{V}\left(\boldsymbol{D}^{T} \boldsymbol{D}+\alpha \boldsymbol{I}\right)^{-1} \boldsymbol{D} \boldsymbol{U}^{T} \boldsymbol{s} .
$$

It was found in the work of Section 2.2 that the above SVD version of the Tikhonov regularization results in more stable solutions than Eq. (15) when $\alpha \rightarrow 0$, and was therefore used in this work.

The $\alpha$ values added to the diagonal elements of $\boldsymbol{D}^{T} \boldsymbol{D}$ in Eq. (16), stabilizes the solution of $\boldsymbol{r}_{\alpha}$ by improving the condition number of the matrix to be inverted, which is now [20]

$$
C(\tilde{\boldsymbol{L}}+\alpha \boldsymbol{I})=\frac{d_{\max }^{2}+\alpha}{d_{\min }^{2}+\alpha} .
$$

It can be seen that as $\alpha \rightarrow 0$, Eq. (15) approaches its original form of Eq. (11) and a more accurate solution should be reached. This is, however, at the cost of numerical stability of the solution as can be seen from Eq. (17) which approaches Eq. (13) when $\alpha \rightarrow 0$, so that a compromise between an accurate solution for $\boldsymbol{r}_{\alpha}$ with small $\alpha$, lying close to an erratic solution (i.e. ill-conditioned), and a less accurate solution for $\boldsymbol{r}_{\alpha}$ with larger $\alpha$, being more stable (i.e. well-conditioned), exists (cf. [17,21]).

If $C_{L 2 S}$ is not known, Eq. (16) can be rewritten as

$$
C_{L 2 s} \boldsymbol{r}_{\alpha}=\boldsymbol{V}\left(\boldsymbol{D}^{T} \boldsymbol{D}+\alpha \boldsymbol{I}\right)^{-1} \boldsymbol{D} \boldsymbol{U}^{T} \boldsymbol{s}
$$

and $\boldsymbol{r}_{\alpha}$ can be obtained by normalizing the solution obtained on the right hand side of Eq. (18) with respect to its maximum component, i.e. by dividing by $\max \left(C_{L 2 s} \boldsymbol{r}_{\alpha}\right)$.

The optimal value of $\alpha$ can be determined by visual inspection of the results (visual inspection is also used by Wing [15] to determine the best solution) - the value of $\alpha$ can be continually decreased up to the point where the solution starts to appear erratic; the optimal $\alpha$ value would be that used in obtaining the solution that just precedes the start of the erratic behaviour. Two noticeable, more formal methods for optimal $\alpha$ value determination are that of the Discrepancy Principle, which connects the $\alpha$ determination with the error level of the data (if known) $[17,19,22]$, and the L-curve method [17,22], which requires no knowledge of the error/noise level of the data. In the L-curve method, solutions must first be calculated for a range of $\alpha$ values, $\alpha_{k}$, with $k$ the index referring to a specific $\alpha$ value in the range, so that a range of solutions $\boldsymbol{r}_{\alpha_{k}}$ is obtained. The values of the logarithm of the residual norm $\left\|C_{L 2 s} \boldsymbol{L} \boldsymbol{r}_{\alpha_{k}}-\boldsymbol{s}\right\|$, appearing in the first term of Eq. (14), is plotted against the logarithm of the solution norm $\left\|C_{L 2 s} \boldsymbol{r}_{\alpha_{k}}\right\|$, appearing in the second term of Eq. (14), which then typically forms an L-shaped curve. This L-curve displays the compromise between the minimization of the residual norm (good LS solution) and the solution norm (damping of instabilities). The optimal $\alpha$ value is the specific $\alpha$ value used in the calculation of the data point situated in the corner of the L-curve, defined as the point on the curve that has maximum curvature. The selection of the corner data point on the L-curve can be done manually or an attempt can be made to calculate its position - a discussion by Hansen on the difficulties involved in computing the point of maximum curvature can be found in [22]; the manual method is used and illustrated in the subsequent sections.

\subsection{Spectral response recovery from modelled measurements}

The following aspects/parameters are controllable during the design and setup of the measurement equation, Eq. (9), and the application of the penalized LS solution, Eq. (15) (and the subsequent SVD implementation thereof as given in Eq. (16)), for resolving the spectral recovery:

1. The number of temperature measurements, $m\left(T_{i}\right.$, with $\left.i=1 \ldots m\right)$, or equivalently the temperature difference $\Delta T$ between temperature values over a given temperature range. The temperature difference, however, needs not to be constant.

2. The temperature range covered by the measured $T_{i},\left[T_{1}, T_{m}\right]$. 
3. The number of wavelength nodes, $n\left(\lambda_{j}\right.$, with $\left.j=1 \ldots n\right)$, or equivalently the wavelength difference $\Delta \lambda$ between equally spaced wavelength values. The wavelength difference needs to be constant when the Trapezoidal quadrature rule is used in the numerical integration.

4. The wavelength range/band covered by $\lambda_{j},\left[\lambda_{1}, \lambda_{n}\right]$, over which the blackbody spectrum is modelled.

5. The value of $\alpha$.

The first two parameters are measurement parameters which must be decided on during the planning of a measurement session, while the following two parameters are modelling parameters which must be fixed before it is attempted to resolve the spectral response with the technique outlined in Section 2.1. The last parameter, $\alpha$, is the solution regularization parameter which must also be fixed before the actual application of Eq. (16). The dependency of the technique on these parameters will now be demonstrated by making use of modelled 'synthetic', noise free values for $\boldsymbol{s}$ containing no measurement uncertainties. These values are obtained by first calculating $\boldsymbol{L}$ in Eq. (8) by using Eq. (3) and the weights prescribed by the Trapezoidal rule, after which $\boldsymbol{L}$ is multiplied an arbitrarily designed normalized response spectrum $\boldsymbol{r}$ to obtain $\boldsymbol{L} \boldsymbol{r}$. In order to obtain the right hand side of Eq. (9), the value of $C_{L 2 s}$ is chosen to be $C_{L 2 s}=1 / \max (\boldsymbol{L r})$ so that vector $\boldsymbol{L r}$ is normalized by its maximum value with the convenient effect that the components of $\boldsymbol{s}$ is scaled between zero and one over the covered temperature range. Note the implicit assumption that $s$ is directly proportional to $L_{p n}$. For these, and all other calculations in this work, the Matlab ${ }^{\circledR}$ software package (version 9.0.0.341360 R2016a) was used on a 64 bit personal computer. The aspects influencing the calculation of $\boldsymbol{L}$ and the impact thereof on the exact values of $L_{p n}$ as obtained from $\boldsymbol{L} \boldsymbol{r}$ will firstly be discussed, before it is attempted to resolve $\boldsymbol{r}$ from only $\boldsymbol{L}$ and the fabricated $\boldsymbol{s}$.

Fig. 1 shows the blackbody spectra $L_{B B}(T, \lambda)$, calculated from Eq. (3), for a set of $m=14$ temperatures $\mathrm{T}=$ $\{100,200, \ldots, 1400\}^{\circ} \mathrm{C}$. The modelled low resolution spectra, with $n=m=14$ wavelength nodes having resolution of $\Delta \lambda \approx 0.9 \mu \mathrm{m}$, compared to the high resolution spectra with $n=116$ wavelength nodes with resulting resolution of $\Delta \lambda=0.1 \mu \mathrm{m}$, clearly show the deviation of the lower resolution spectrum from the more accurate, smoother high resolution spectra. This deviation is especially obvious close to the peak value associated with each blackbody temperature (described by Wienn's displacement law). It should therefore come as no surprise that the areas under the respective curves might differ and the integral of the high resolution curve would be more accurate than the integral of the low resolution curve for a blackbody of specified temperature. The implication is that the value of $n$, and therefore the value of $\Delta \lambda$, would have an impact on the calculation of a numerical integral of $L_{b b}$ in Eq. (3) over a specified wavelength range. The value of $n$ therefore impacts on the calculation of the accuracy of the numerical integral given by Eq. (5) - the parameter $n$ should be large enough in order to model the measured signal with adequate accuracy.

Fig. 2 shows the peak-normalized $3-5 \mu \mathrm{m}$ band radiance modelled 'calibration' curves for different values of $m$, with $n=m$ in each instance, calculated with Eq. (5) for a normalized spectral responsivity $r(\lambda)$ having a flat spectral response with value of one over $3-5 \mu \mathrm{m}$ and zero response outside this region (this spectral block response is shown in the graphs of Fig. 4 by a thick black curve). The smoothness of each curve increases as the number of data points defining the curve, i.e. the number of chosen temperatures $m$ in the $100-1400{ }^{\circ} \mathrm{C}$ temperature range, increases - the least smooth curve is for $m=11$, while the smoothest curve is for $m=1301$. Furthermore, as noted in the previous paragraph, the accuracy of the calculations using Eq. (5) increases as the number of wavelength nodes $n$ increases, so that, with $n=m$, the accuracy of each curve increases as the number of temperatures $m$ increases. It can be seen that as $m$ increases, the different curves converge towards and settles on the curve with the highest value of $m$, i.e the curve with 1301 temperature values with $\Delta T=1^{\circ} \mathrm{C}$ and an accompanying number of 1301 wavelength nodes.

It should be noted that the accuracy of the curves in Fig. 2 is, besides being influenced by $m$, also influenced by the specific wavelength range/band of interest as covered by $\left[\lambda_{1}, \lambda_{n}\right]$ and as imposed by $r(\lambda)$, since for the same $n$ and $\Delta \lambda$ used in the calculations, a calculated value of $L_{p n}\left(T_{i}\right)$ at a specific $T_{i}$ can be more accurate in one wavelength range than in another, e.g. the calculated $L_{p n}$ at a high value of $T_{i}$ is more accurate in the $3-5 \mu \mathrm{m}$ band than in the $1-3 \mu \mathrm{m}$ band. This is due to the larger approximation errors (as touched on earlier) of the numerical integral around the peak radiance values, which are situated in $1-3 \mu \mathrm{m}$; this can easily be gathered by comparison of the solid and dotted blackbody curves for high temperatures show in Fig. 1 in this band. It can be said that above some value of $n(=m)$, the values of $L_{p n}$ would be adequately modelled in a specific wavelength band for a specific response spectrum. 


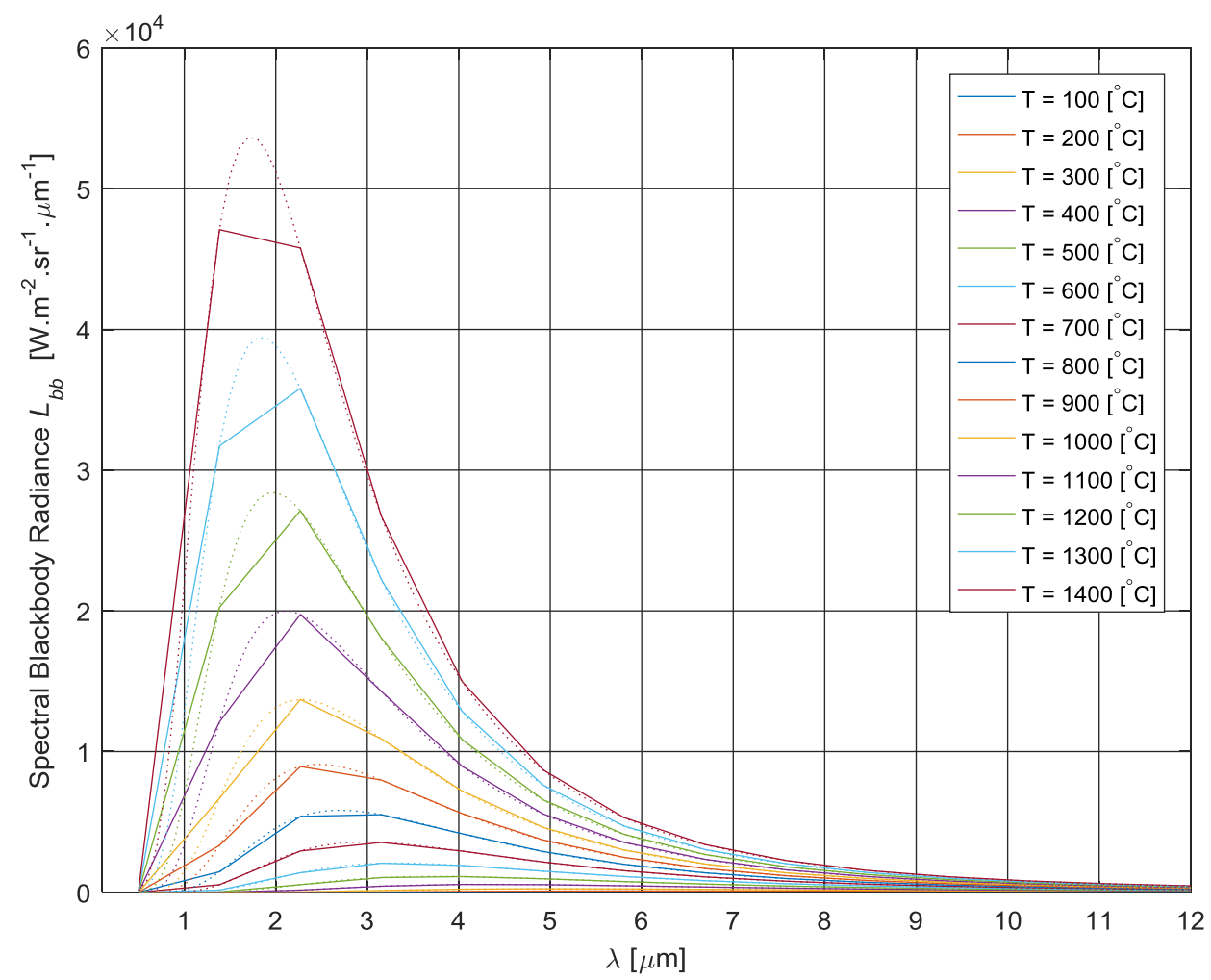

Fig. 1. Illustration of blackbody spectra over $0.5-12 \mu \mathrm{m}$ for different temperatures. It can be seen how the low resolution spectra with $n=14$ and $\Delta \lambda=0.9 \mu \mathrm{m}$ (solid lines) deviates from the more accurate high resolution spectra $n=116$ and $\Delta \lambda=0.1 \mu \mathrm{m}$ (dotted lines), especially around the peak of each spectrum associated with the higher temperatures.

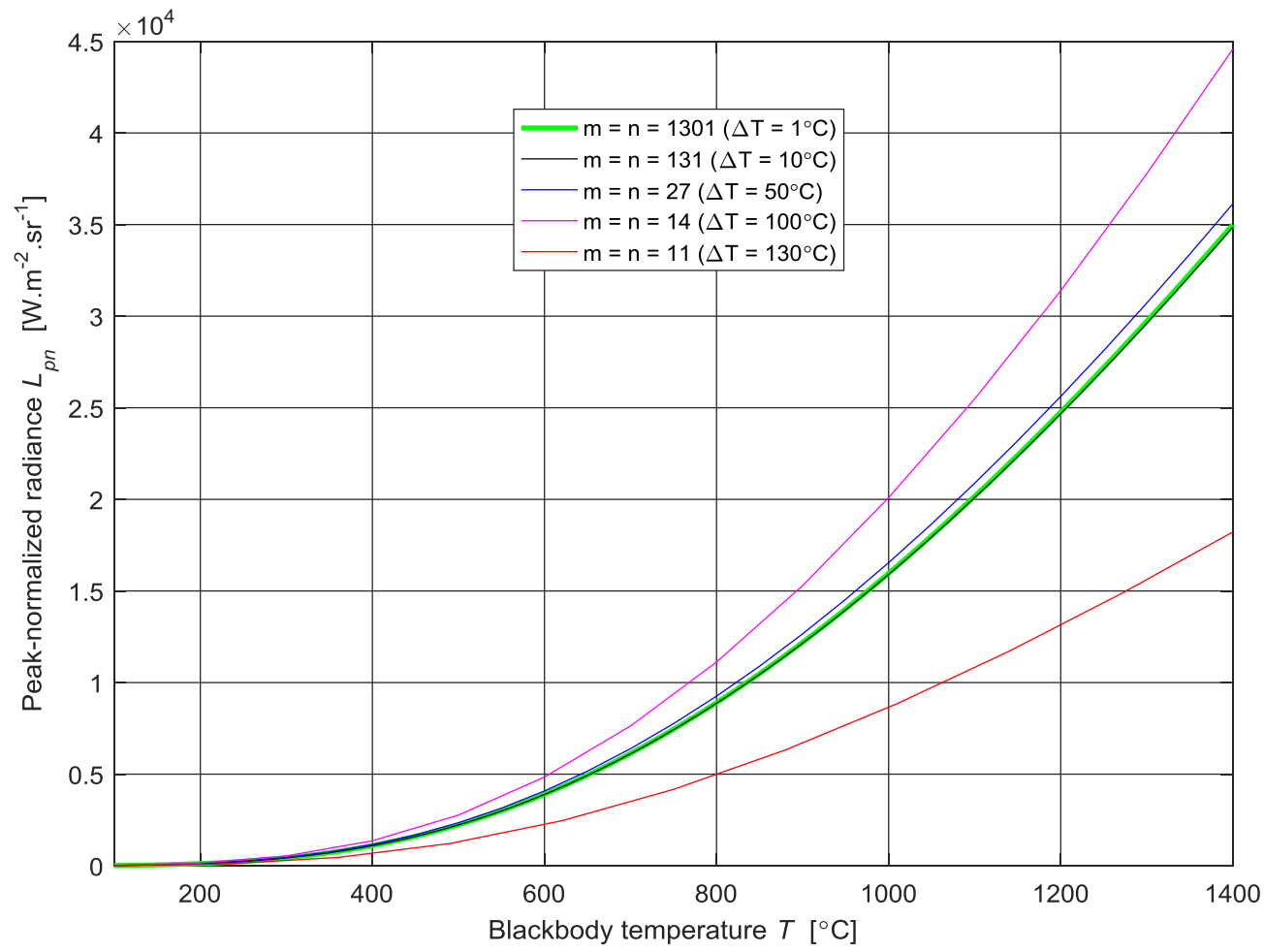

Fig. 2. The peak-normalized radiance as a function of blackbody temperature for the block spectral response over $3-5 \mu \mathrm{m}$ shown in Fig. 4, i.e. calibration curves. The curves were calculated using different numbers of blackbody temperatures, $m$, and wavelength nodes, $n$, although $n$ was chosen so that $n=m$. Only straight line interpolation between data points was used in plotting these curves. 
Fig. 3 shows the relationship between the modelled $s$ and $L_{p n}$ values as calculated by Eq. (6) for the $3-$ $5 \mu \mathrm{m}$ block response and temperature values used in Fig. 1 for the case when $m=14$ (with $n=m$ ), i.e. for $T=\{100,200, \ldots, 1400\}^{\circ} \mathrm{C}$. The relationship is ideal in the sense that $s$ is directly proportional to $L_{p n}$, as can be gathered from Eqs. (6) and (9), with no noise threshold or any non-linearities present in $s$. For cases where $m>14$, as will be used later in resolving $\boldsymbol{r}$, the gradient of the straight line fit to the data points will be different to the one shown in the figure due to the improved accuracy of the calculated $L_{p n}$ values.

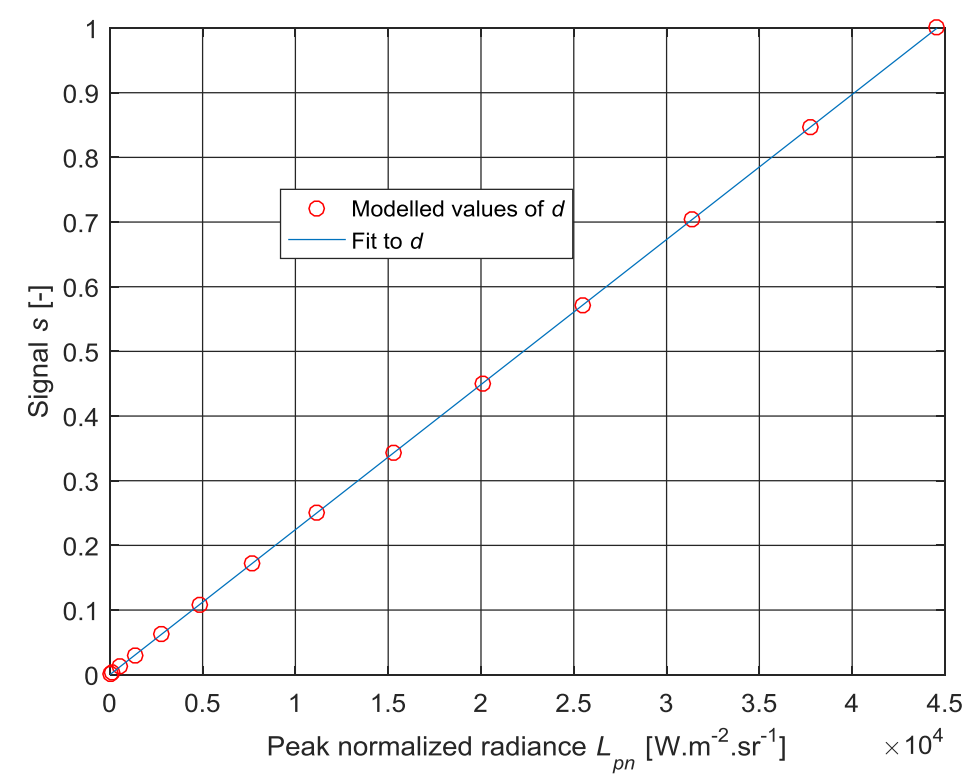

Fig. 3. The modelled signal values, $s$, resulting from the peak normalized radiance, $L_{p n}(T)$, at different blackbody

temperatures.

Having gained some insight into the importance of the role played by the measurement parameter $m$ (and the associated $\Delta T$ ) and the modelling parameters $n$ (with associated $\Delta \lambda$ ) and wavelength band $\left[\lambda_{1}, \lambda_{n}\right]$ in setting up the measurement equation given by Eq. (9), the dependency of the recovery technique, as condensed by Eq. (16) or Eq. (18), on these as well as the remaining parameters of $\alpha$ and temperature range $\left[T_{1}, T_{m}\right]$, will now be demonstrated; in this respect, Fig. 4 shows the results (to be discussed) of various spectral response recoveries in which different values of the mentioned parameters were used, given only the $\boldsymbol{S}$ and $\boldsymbol{L}$ values (as calculated from the mentioned $3-5 \mu \mathrm{m}$ block response) as input. Although the value of $C_{L 2 s}$ was considered to be unknown, the value of $\boldsymbol{r}_{\alpha}$ could be obtained in each instance by normalizing the vector calculated on the right hand side of Eq. (18) by its largest component.

To start off with, calibration measurements were modelled for blackbody spectra over the temperature range $100-1400^{\circ} \mathrm{C}$ with $\Delta T=100^{\circ} \mathrm{C}$. The number of wavelength nodes was set equal to the number of temperatures, i.e. $n=m$, with $m=14$ and therefore $\Delta \lambda \approx 0.9 \mu m$ over a wavelength range of $\lambda=0.5-$ $12 \mu m$ (the applicable calibration curve, with $\Delta T=100^{\circ} \mathrm{C}$ and $m=n=14$, is shown in Fig. 2). The characteristics of the Tikhonov approximation towards the spectral response recovery for these setup parameters can be observed as a function of $\alpha$ in Fig. $4 \mathrm{a}$ - at the relatively large value of $\alpha=1$ the solution is stable, although not that accurate; at the smallest value of $\alpha=0$ (i.e. no regularization), the solution becomes erratic, with extreme values outside the MWIR response wavelength band; over some optimal range of $\alpha$ values, between $\alpha=10^{-19}$ and $\alpha=10^{-21}$ in this case (note that the green coloured curve lies on top of the cyan coloured curve), the resolved response spectrum appears to be reasonable in comparison with the correct spectrum shown in black (in spite of its calibration curve in Fig. 2 seeming less than adequately accurate). The value of $\alpha$ can also be obtained from the $\alpha$ values used in the calculation of the corner point(s) of the L-curve shown in Fig. 5, calculated from $\alpha=10^{0}, 10^{-2}, 10^{-4}, \ldots, 10^{-26}, 10^{-28}$. The values of $\alpha$ for the three points clustered in the corner position of the curve are $\alpha=10^{-18}, 10^{-20}$ and $10^{-22}$, which agrees with the results obtained from the visual inspection. The L-curves obtained for the remainder of the solutions shown in Fig. $4 \mathrm{~b}$ - Fig. $4 \mathrm{f}$ (discussion to follow hereafter) all had optimal $\alpha$ values over this wide range, with small to no distinction that could be made between their solutions. 
Modelling of the measurements of the previous paragraph were repeated over the same blackbody temperature range of $100-1400^{\circ} \mathrm{C}$ but with an increase in the number of temperatures from $m=14$ to $m=131$, with the resultant value of the constant temperature interval decreasing from $\Delta T=100{ }^{\circ} \mathrm{C}$ to $\Delta T=10^{\circ} \mathrm{C}$. The modelling parameter $n$ was again set equal to the now larger value of $m$. Using these 'measurements', the resolved spectra in Fig. $4 \mathrm{~b}$ are now smoother for the three largest values of $\alpha$ due to the higher wavelength resolution, although these spectra are not necessarily better solutions than those obtained in Fig. 4a. A value of $\alpha=1 \times 10^{-21}$ was found to give a relatively good recovery of the response spectrum, although instability is already starting to show. The value of $\alpha=0$ results in a totally erratic response spectrum.

Fig. $4 \mathrm{c}$ shows the recovered response spectra for the same temperature interval of $\Delta T=10{ }^{\circ} \mathrm{C}$ between different blackbody modelled measurements as used for Fig. $4 \mathrm{~b}$, but for a much narrower temperature range of $\mathrm{T}=200-300^{\circ} \mathrm{C}$. The most useable solution was also found at $\alpha=10^{-21}$. Although the wavelength resolution of the spectrum is not ideal $(n=m=11)$, the recovered response can still be used in determining the wavelength band in which the instrument operates (clearly MWIR in this case). This solution is, however, not as good as that obtained in Fig. 4a and Fig. 4b.

In Fig. $4 d$, the same temperature range and interval was used than in Fig. $4 \mathrm{~b}$, but with $n \approx \frac{1}{2} m$ (now an overdetermined system of measurement equations). Although the wavelength resolution is half that than for Fig. $4 \mathrm{~b}$, the quality of the best recovered response spectra $\left(\alpha=10^{-19}-10^{-21}\right)$ appears to be similar than that of Fig. $4 \mathrm{~b}$.

The parameter values used in obtaining the recovered spectra shown in Fig. 4e are the same as those used for Fig. $4 \mathrm{~b}$, except for the wavelength range which was chosen to be relatively narrow around the MWIR band, i.e. $\lambda=2.5-5.5 \mu \mathrm{m}$, and the resulting smaller value of $\Delta \lambda \approx 0.023 \mu \mathrm{m}$ (i.e. higher wavelength resolution). It can be seen that the recovered response spectrum in Fig. $4 \mathrm{e}\left(\alpha=10^{-19}-10^{-21}\right)$ agrees more closely to the correct spectrum than that shown in Fig. $4 \mathrm{~b}$.

The spectra shown in Fig. 4f were obtained by increasing the temperature resolution used for Fig. 4e by setting $\Delta T=2{ }^{\circ} \mathrm{C}$ with a resulting change from $\Delta \lambda \approx 0.023 \mu \mathrm{m}$ to $\Delta \lambda \approx 0.005 \mu \mathrm{m}$. Comparison of the recovered high resolution spectra of Fig. $4 \mathrm{f}$ and the lower resolution spectra of Fig. $4 \mathrm{e}$ yields little difference between them, as was the case in the comparisons of Fig. 4b with Fig. 4a and Fig. 4b with Fig. 4d.

The spectral recovery of synthetically generated data is now further explored in order to illustrate that the technique is also able to recover spectra of more complex shapes than that of the $3-5 \mu \mathrm{m}$ block response used so far.

From Eq. (5) it is clear that due to the dependency of $L_{p n}(T)$ on $r(\lambda)$, different shapes of $r(\lambda)$ should result in different $L_{p n} v s . T$ curves. Fig. $6 \mathrm{~b}$ illustrates the calibration curves of peak-normalized radiance calculated from Eq. (5) as a function of blackbody temperature (similar to curves in Fig. 2) covering $T=50-500{ }^{\circ} \mathrm{C}$ with $\Delta T=10^{\circ} \mathrm{C}$ as modelled for the different instrument spectral responses shown in Fig. 6a. These calibration curves (Fig. 6b) differ from each other as a result of the mentioned differences in the spectral responses - note how some curves cross each other, indicating that these curves do not just differ by a scaling factor.

Using an adequate temperature and wavelength range and resolution (cf. the discussion on the curve accuracies in Fig. 2), the spectral response should be resolvable from the curves of Fig. 6. In order to demonstrate this claim, the resolved normalized response spectrum of each of these instrument response curves are shown in Fig. 7. In each case, the wavelength range was chosen as to tightly fit around the known response spectrum. This can always be done for any unknown $r(\lambda)$ by firstly recovering the spectrum over a wide wavelength range, as shown in Fig. 4a-d, and then narrowing down on the wavelength region in which $r_{\alpha}(\lambda)$ is shown to have a non-zero response, as was done in Fig. 4e-f. The optimal values of $\alpha$ were obtained from the method of visual inspection as well as the L-curve procedure. 
(a)

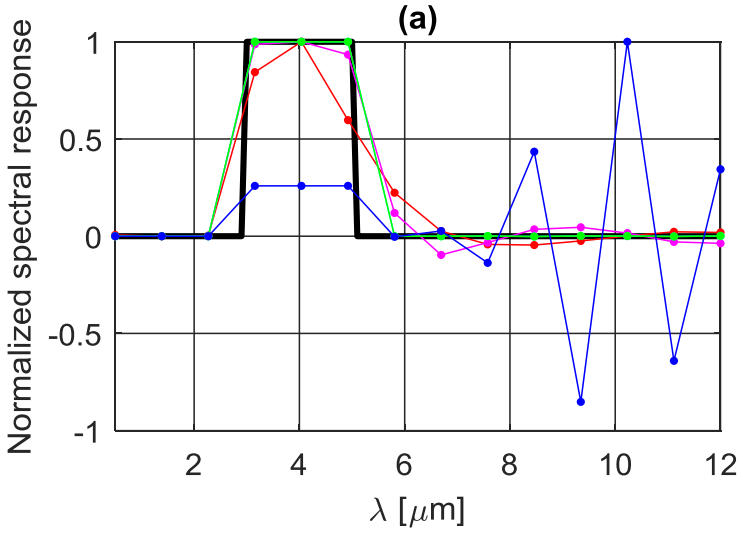

(c)

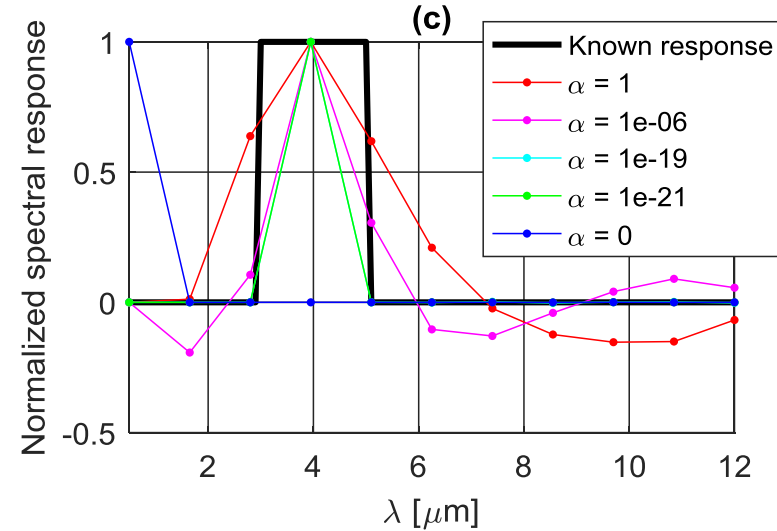

(e)

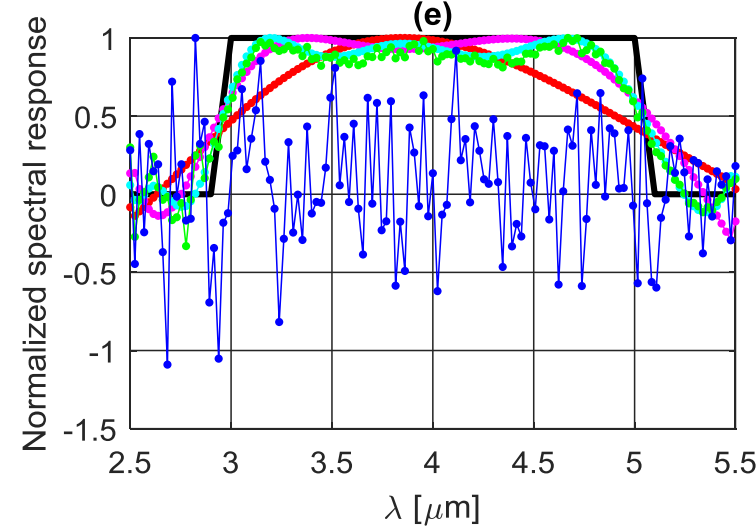

(b)

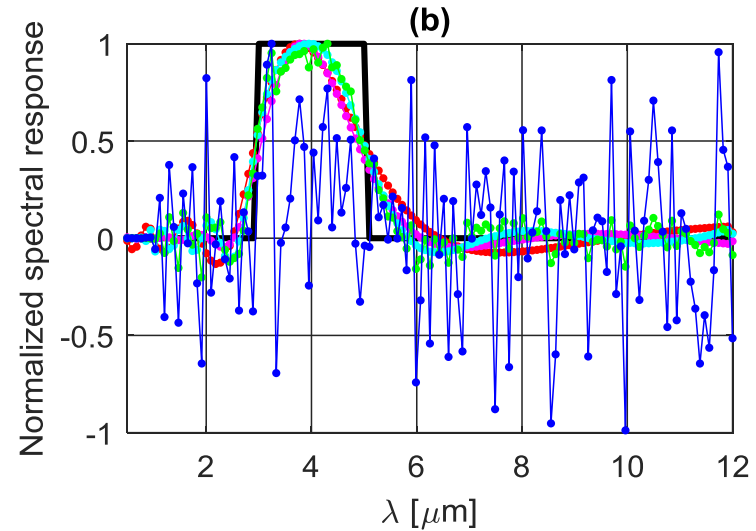

(d)

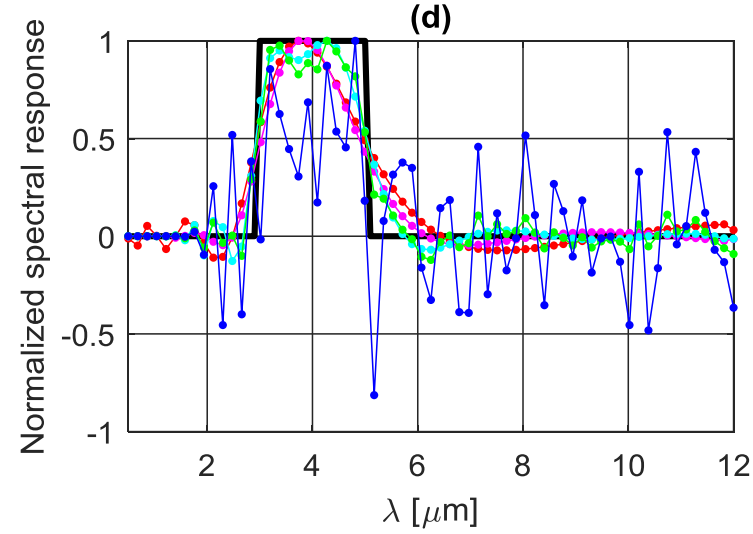

(f)

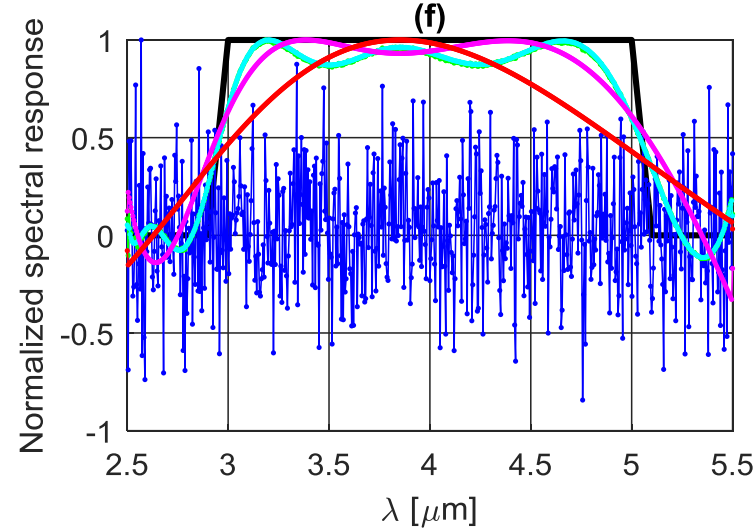

Fig. 4. The correct spectral response, $r(\lambda)$, used in the calculation of the instrument output signal, $s$, is shown by thick black curves together with the resolved response spectra, $r_{\alpha}$, obtained by using different values of $\alpha$ for modelled blackbody measurements with

a) $\Delta T=100{ }^{\circ} \mathrm{C}$ covering the range $100-1400^{\circ} \mathrm{C}$, the number of wavelength nodes equal to the number of temperatures, i.e. $n=m=14$ and therefore $\Delta \lambda \approx 0.885 \mu m$ over a wavelength range of $\lambda=0.5-12 \mu m$. Note that cyan and green curves lie on top of each other.

b) $\Delta T=10^{\circ} \mathrm{C}$ covering the range $100-1400^{\circ} \mathrm{C}$, with $n=m=131, \Delta \lambda \approx 0.088 \mu \mathrm{m}$ over $\lambda=0.5-12 \mu \mathrm{m}$.

c) $\Delta T=10^{\circ} \mathrm{C}$ covering the range $200-300^{\circ} \mathrm{C}$ with $n=m=11, \Delta \lambda \approx 1.15 \mu \mathrm{m}$ over $\lambda=0.5-12 \mu \mathrm{m}$. Note that cyan and green curves lie on top of each other.

d) $\Delta T=10^{\circ} \mathrm{C}$ covering the range $100-1400^{\circ} \mathrm{C}$ with $n=65 \approx \frac{1}{2} m, \Delta \lambda \approx 0.18 \mu \mathrm{m}$ over $\lambda=0.5-12 \mu \mathrm{m}$.

e) $\Delta T=10^{\circ} \mathrm{C}$ covering the range $100-1400^{\circ} \mathrm{C}$ with $n=m=131, \Delta \lambda \approx 0.023 \mu \mathrm{m}$ over $\lambda=2.5-5.5 \mu \mathrm{m}$.

f) $\Delta T=2{ }^{\circ} \mathrm{C}$ covering the range $100-1400^{\circ} \mathrm{C}$ with $n=m=651, \Delta \lambda \approx 0.005 \mu \mathrm{m}$ over $\lambda=2.5-5.5 \mu \mathrm{m}$. Note that cyan and green curves lie on top of each other. 


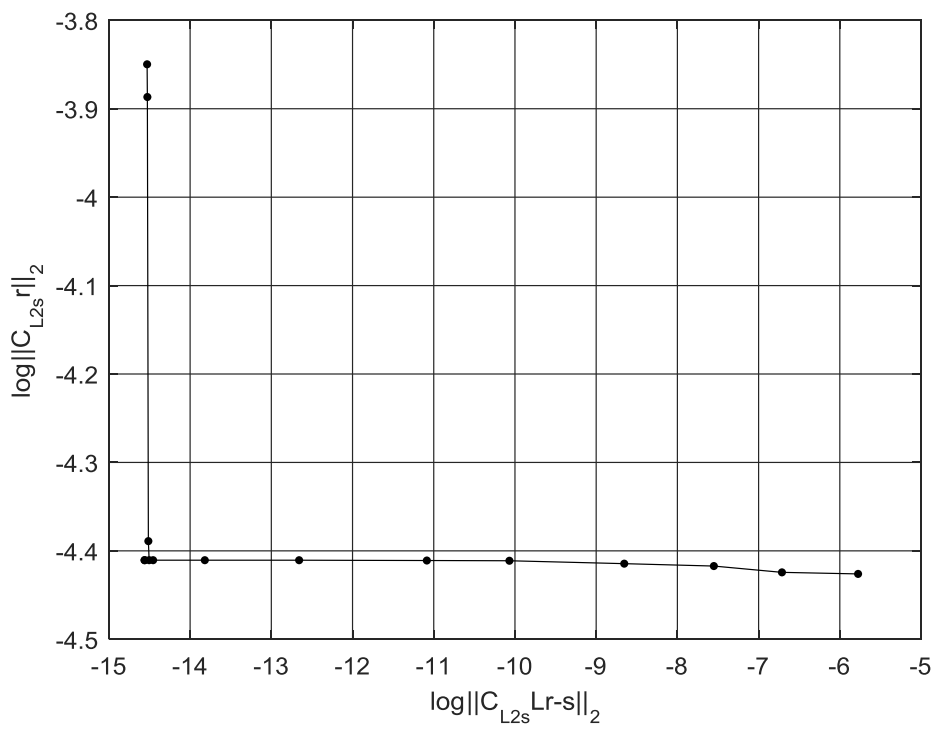

Fig. 5. The L-curve of the solutions shown in Fig. 4a. The $\alpha$ value(s) used for the calculation of the data points in the corner of the curve give the optimal value(s) of $\alpha$. The $\alpha$ values used in the calculation of the curve was $\alpha=$ $10^{0}, 10^{-2}, 10^{-4}, \ldots, 10^{-26}, 10^{-28}$. 
(a)
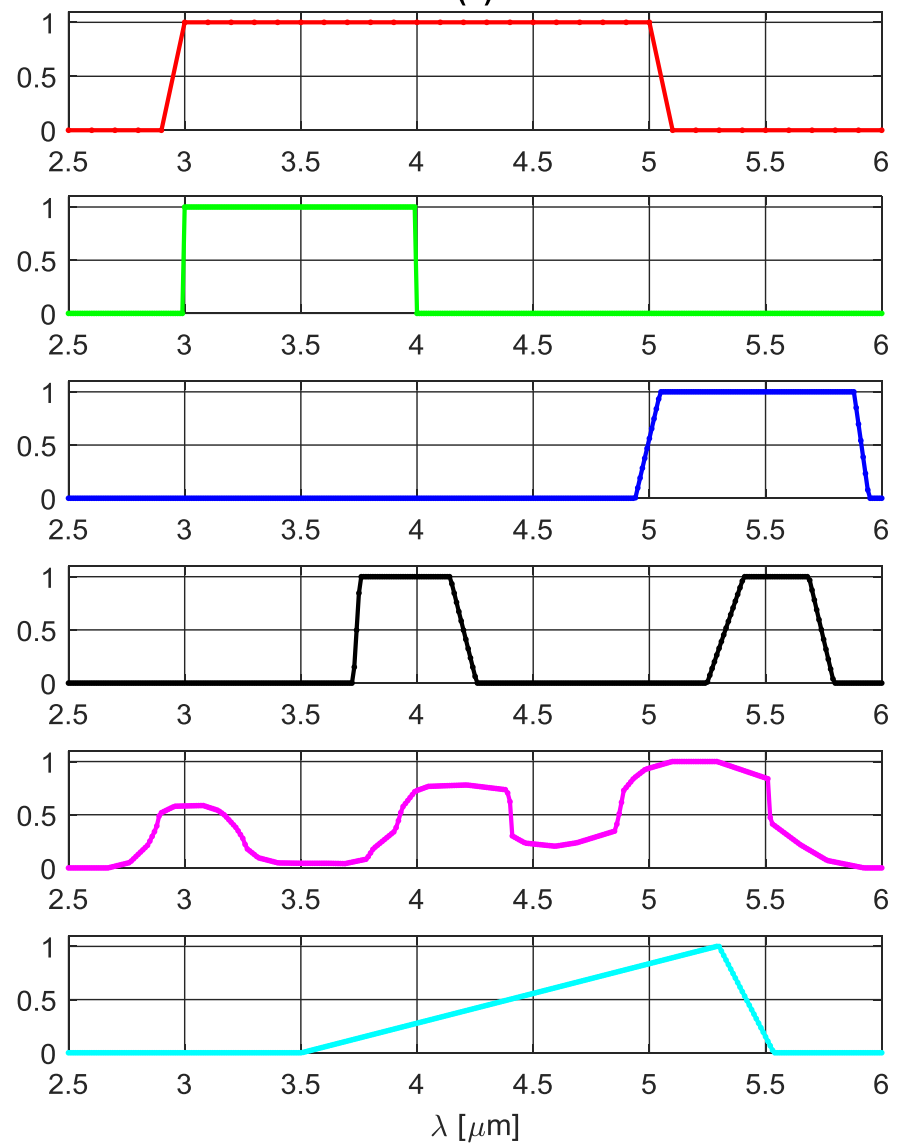

(b)

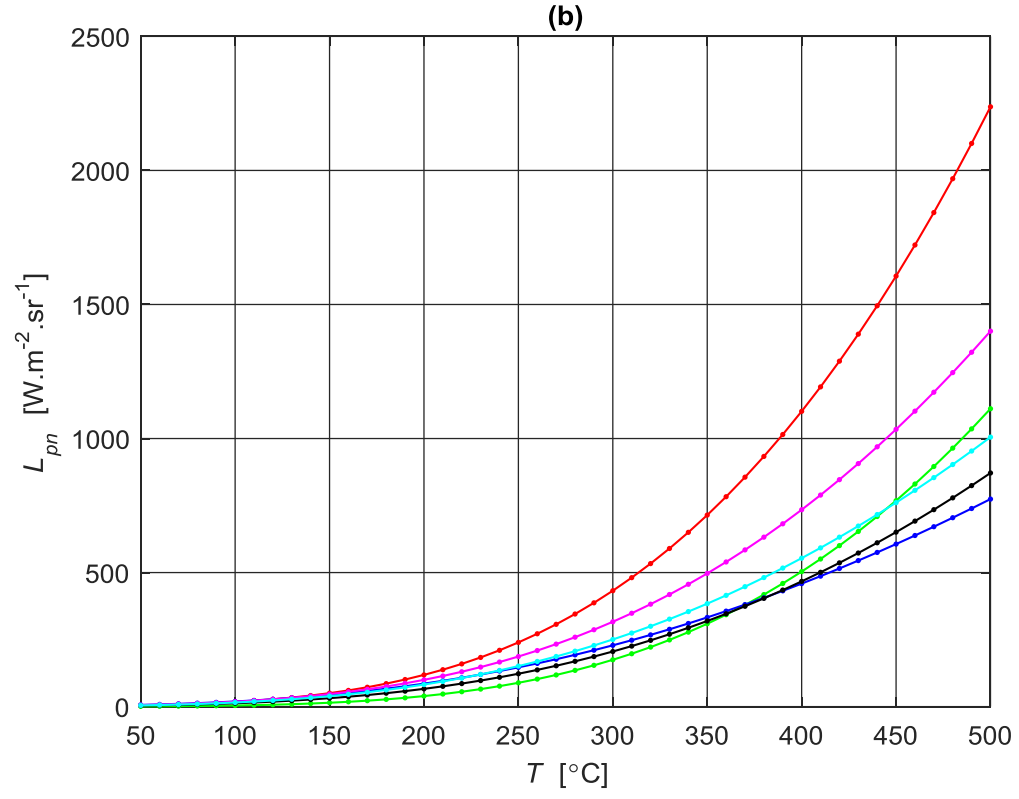

Fig. 6. The (b) modelled peak-normalized radiance, $L_{p n}(T)$, as a function of blackbody temperature, $T$, as calculated from (a) different normalized response spectra $r(\lambda)$. The colours and line types of the spectra in (b) agrees with the colours used in (a). 
(a)

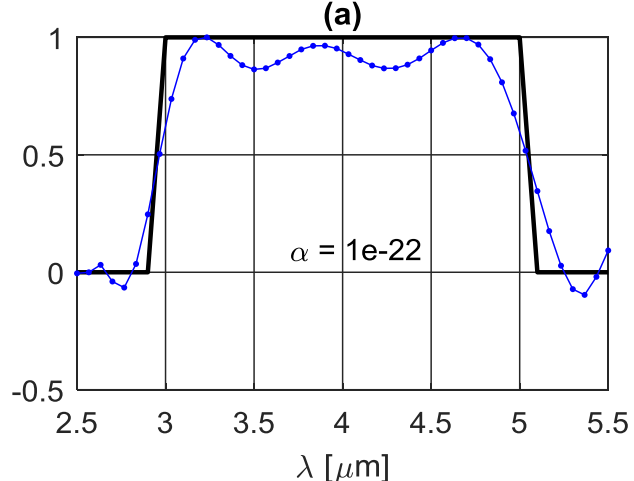

(c)

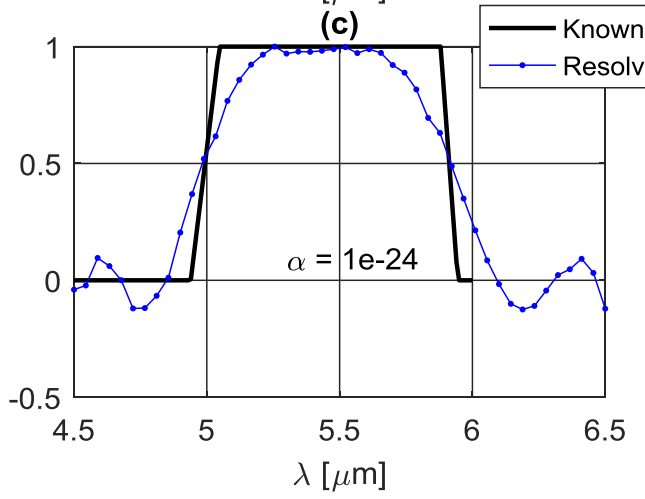

(e)

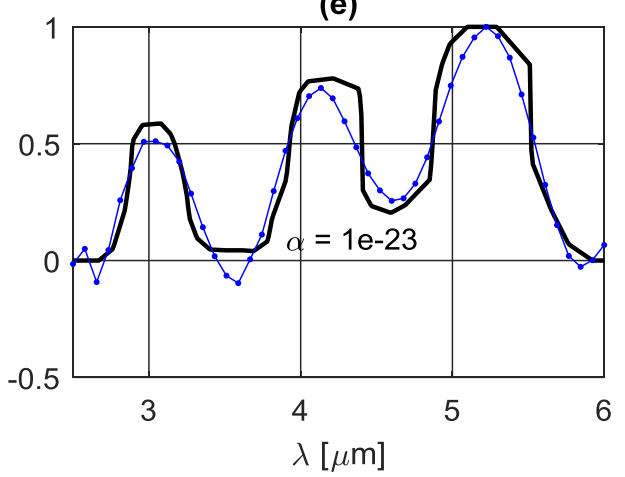

(b)

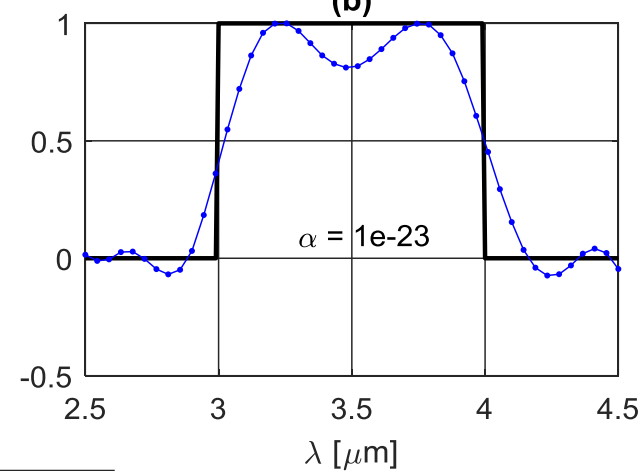

(d)

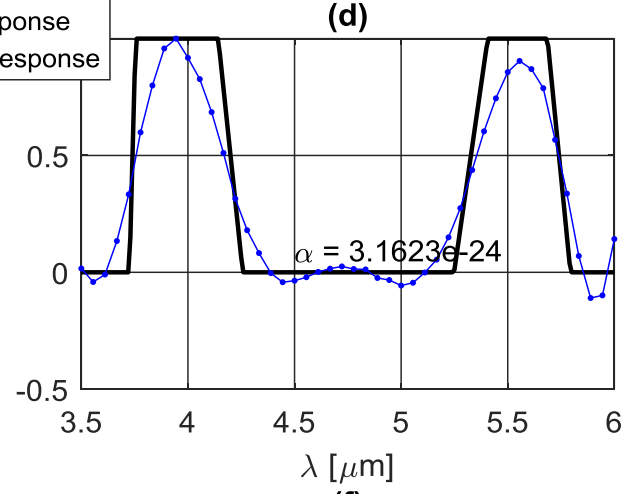

(f)

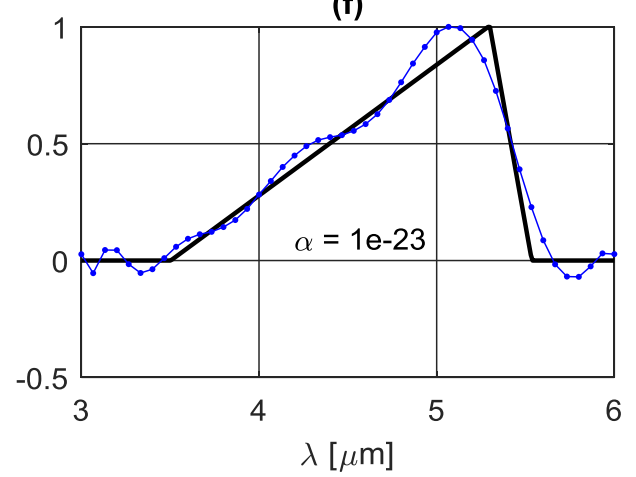

Fig. 7. The resolved normalized spectral response of the various, arbitrary response spectra shown in Fig. 6 , for $T=$ $50-500{ }^{\circ} \mathrm{C}$ and $\Delta T=10^{\circ} \mathrm{C}$. The number of wavelength nodes was chosen such that $n=m$.

\subsection{Insights gained from applying the technique to the modelled measurements}

During the application of Tikhonov regularization in Eq. (16) to the data as modelled by Eq. (9), the value of the regularization parameter $\alpha$ was obtained on the basis of visual inspection of the computed solution as well as from the L-curve selection method. During the visual inspection method, the order of magnitude of the smallest value of $\alpha$, which is supposed to give the best representation of the actual response function, was easily (and quickly) obtained by calculating the solution $r_{\alpha}(\lambda)$ for a decreasing series of $\alpha$ values. The value of $\alpha$ to be used is that which almost results in numerical instability of the computed solution - this could just as easily be seen in the results of Fig. 4. Changes to the value of $\alpha$ within the correct order of magnitude do typically not result in any critical changes to the computed solution, so that the visual inspection method with systematic experimentation with the $\alpha$ value is a viable method of resolving the response spectrum.

Determination of the optimal value of $\alpha$ using the L-curve method resulted in answers which were in good agreement with the visual inspection method. In some cases, more than one data point cluttered together in the corner of the L-curve. The use of the $\alpha$ values of these cluttered data points resulted in recovered spectra which were mostly indistinguishable from each other, thus giving a range of optimal $\alpha$ values.

Although the study in Section 2.2 on the behaviour of the applied technique with respect to the different measurement and modelling parameters was not exhaustive, some important insights could still be gained: 
- The number of wavelength nodes $n$ should be large enough to enable accurate modelling of $L_{p n}$ in the measurement equation, Eq. (9). It would therefore be good practice to set $n$ equal to the maximum value for which the system of measurement equations is not underdetermined, i.e. a square system with $n=m$. The implication is that the number of temperatures $m$ should be sufficient to allow accurate modelling of $L_{p n}$.

- Although higher values of $n$ results in a recovered response spectrum with higher wavelength resolution, it does not guarantee the increased stability of the solution.

- A wider range of temperature values resulted in a better solution of the response spectrum, so that it is advisable to cover the widest possible temperature range that can be measured.

- Since complex shapes generally require more data points to describe them, complex response spectra can only be described by spectral response data with sufficiently high wavelength resolution, specified by $n$. However, if $n$ is too large (and therefore $\Delta \lambda$ too small), $L_{i, j}$ and $L_{i, j+1}$ become very close in value, resulting in columns $j$ and $j+1$ of matrix $\tilde{\boldsymbol{L}}$ to be nearly equal. These nearly equal columns cause $\tilde{\boldsymbol{L}}$ to be almost singular, and $\tilde{\boldsymbol{L}}^{-\mathbf{1}}$ can be expected to exhibit poor behaviour [15]. Other quadrature schemes than the Trapezoidal method, possibly better fitted to the combination of blackbody curve and expected shape and region covered by the response spectrum, can be used and might improve the numerical results [15].

- The modelling wavelength range should fit tightly around the expected wavelength region covered by the response spectrum. If this region is unknown, the calculations can be started over a wide range, and then repeated over a narrower range after the region of active spectral response has been determined.

Resolving the IFK in Eq. (4) not only requires a regularization method, but also subject knowledge regarding the problem at hand. In this respect, it is known that the normalized spectral responsivity is bounded by $0 \leq r(\lambda) \leq 1$. Finalization in resolving $r_{\alpha}(\lambda)$, e.g. the spectra shown in Fig. 4 and Fig. 7, can be obtained by imposing responsivity boundaries of $r_{\alpha}(\lambda)=0$ for all negative values of $r_{\alpha}(\lambda)$. Furthermore, from knowledge of the detector material (e.g. InSb or $\mathrm{HgCdTe}$ ) when working with a real instrument and qualitative transmission properties of the optical system (e.g. knowledge of the infrared band for which the lens was manufactured, e.g. LWIR), it should be known whether only one (Fig. 7a,b,c,f) or several (Fig. 7d,e) wavelength regions of non-zero spectral responsivity exist. If only one region exists, then all $r_{\alpha}(\lambda)>0$ can be set to zero in wavelength regions suspected not to partake in the instrument responsivity. In order to determine whether the adjustments to $r_{\alpha}(\lambda)$ during finalization of the resolution has not wrecked the true response $r(\alpha)$, the left hand side of Eq. (9) can be calculated using $r_{\alpha}(\lambda)$, i.e. $\boldsymbol{L} \boldsymbol{r}_{\boldsymbol{\alpha}}$, and its relationship with the measured $\boldsymbol{s}$ can be tested (as in Fig. 3) to determine whether the expected linear dependency exists. Any major deviation from this assumed linear relationship would indicate an inadequately resolved responsivity. Care must however be taken if it is known that the instrument has such a nature that the output signal varies non-linearly with the observed radiance. In such a case, the non-linearity must first be characterized and then an attempt can be made to linearize the output signal $[23,24]$, after which the outlined technique can possibly be applied.

\section{DETERMINING THE SPECTRAL RESPONSE OF AN INFRARED CAMERA}

The spectral response for a (2003 model) Cedip Jade III MWIR camera, our camera under investigation (CUI), with a cooled InSb SCD Gemini detector was determined using the three methods mentioned in Section 1, i.e. theoretically, purely experimentally and by using the technique outlined in Section 2.1. This camera has an image resolution of $320 \times 240$ pixels with a field of view (FOV) of approx. $11^{\circ} \times 8^{\circ}$ obtained by using a $50 \mathrm{~mm}$ lens with an f-number of $f / \#=2$.

\subsection{Theoretically and purely experimentally}

Theoretically, the spectral responsivity for this instrument can be written as

$$
\Re_{\text {instr }}(\lambda)=\Re_{\text {detector }}(\lambda) \tau_{\text {dewar }}(\lambda) \tau_{\text {ar }}(\lambda) \tau_{\text {lens }}(\lambda)
$$

where $\mathfrak{R}_{\text {detector }}(\lambda)=k \eta(\lambda) \lambda$ is detector responsivity, $k$ is a constant, $\eta$ is the spectral quantum efficiency and $\tau_{\text {dewar }}(\lambda), \tau_{a r}(\lambda)$ and $\tau_{\text {lens }}(\lambda)$ are the spectral transmissions of the dewar window, anti-reflection 
coating on the dewar window and the lens respectively. The normalized detector spectral responses would then be

$$
r_{\text {detector }}(\lambda)=\frac{\Re_{\text {detector }}(\lambda)}{\Re_{\text {detector }}\left(\lambda_{d}\right)}
$$

and the normalized instrument response is

$$
r_{\text {instr }}(\lambda)=\frac{\Re_{\text {instr }}(\lambda)}{\Re_{\text {instr }}\left(\lambda_{0}\right)}
$$

where $\mathfrak{R}_{\text {detector }}(\lambda)$ has a maximum value at $\lambda_{d}$ and $\mathfrak{R}_{\text {instr }}(\lambda)$ has a maximum value at $\lambda_{0}$. Typically representative transmission data of $\tau_{\text {dewar }}(\lambda)$ and $\tau_{\text {ar }}(\lambda)$ for this camera model were obtained from the manufacturer and are shown in Fig. 8 together with $r_{\text {detector }}(\lambda)$ and $r_{\text {instr }}(\lambda)$. The silicon lens, with its own anti-reflection coatings, has a flat transmission spectrum in the MWIR region (not shown in the figure).

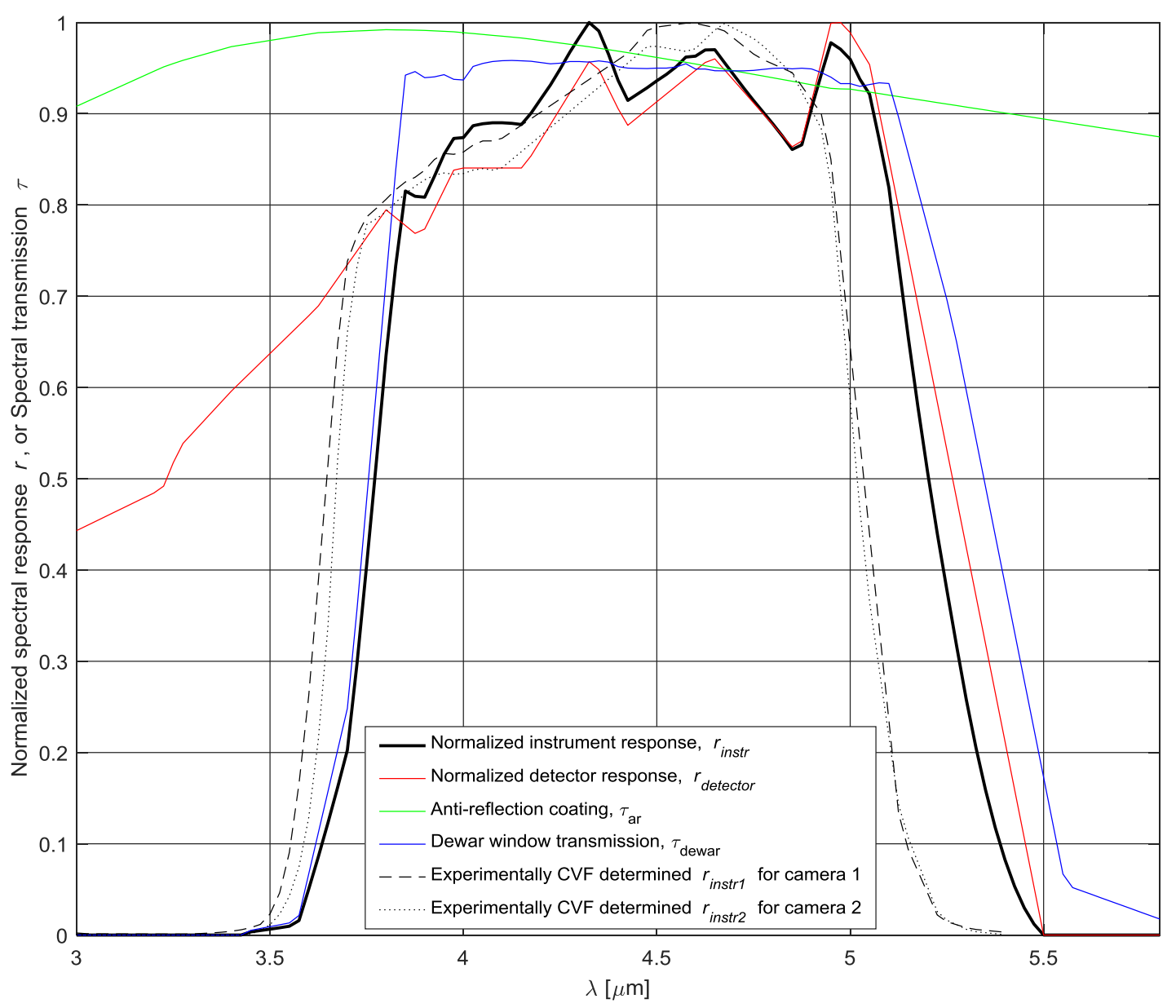

Fig. 8. The spectral transmissions of the optical components $\left(\tau_{a r}\right.$ and $\left.\tau_{\text {dewar }}\right)$ and normalized detector responsivity $\left(r_{\text {detector }}\right)$ of the MWIR camera, together with the normalized instrument response $\left(r_{\text {instr }}\right)$ calculated from these variables and experimentally determined instrument responses $\left(r_{\text {instr } 1}\right.$ and $\left.r_{\text {instr } 2}\right)$ by using a CVF.

The spectral response of two camera models identical to the CUIs, one with a $50 \mathrm{~mm}$ lens $\left(r_{\text {instr } 1}\right)$ and the other with a $100 \mathrm{~mm}$ lens $\left(r_{\text {instr } 2}\right)$, were obtained purely experimentally with the use of a circular variable filter (CVF) wheel (model S134-LR of Optical Coatings Laboratory Inc.) [25]. The spectral responses so obtained are also shown in Fig. 8. It can be seen that these response curves differ from the theoretically 
determined curve, and they also differ from each other (although not much) - this indicates the probable deviations of the manufactured components of the camera from the typical data available on these components. The experimentally determined camera responses will be considered as the most accurate representation of their actual response spectra, with the response spectrum of the camera with the $50 \mathrm{~mm}$ lens that will be used as a reference spectrum to verify any resolved spectrum against.

\subsection{Resolving the instrument response by the proposed technique}

The measurement with an ideal instrument of the infrared radiation from an object of interest, with no influences on the measurement by the environment, was described in Section 2.1. The CUI is, however, a real instrument subjected to influences from its environment and a realistic model of the signal output from the instrument should be constructed before attempting to resolve its spectral responsivity.

\subsubsection{Refinement of the proposed technique for a non-ideal instrument}

When the CUI is observing a blackbody, the measurement equation for the total signal output of the camera, $s_{\text {tot }}$, can be modelled (cf. $\left.[26,27]\right)$ by

$$
s_{\text {tot }}=\frac{d}{d t}\left[s(T)+s_{e}\left(T_{e}\right)+s_{d}\left(T_{d}\right)+\cdots\right] t+\Delta s,
$$

where $s(T)$ is the component of $s_{\text {tot }}$ originating from the observed blackbody radiance (as defined in Section 2.1), $s_{e}\left(T_{e}\right)$ is the component of $s_{t o t}$ originating from the inside environment of the camera which has stabilized at the camera internal temperature, $T_{e}$, and $s_{d}\left(T_{d}\right)$ is the signal component resulting from the detector dark current, which is dependent on the detector temperature, $T_{d}$. The contribution of these components are all dependent on the integration time setting, $t$, of the camera during a measurement. Any possibly remaining integration time dependent signal components are represented by ' $\ldots$ ' in Eq. (22). Any $t$ independent contributions to $s_{t o t}$, like an offset in the readout circuitry, is represented by $\Delta s$. The influence of the outside environment is considered to be limited to the blackbody radiance when observed over a small distance, so that atmospheric radiation (path radiance) and attenuation are assumed to be negligible.

If, before the start of a set of measurements, the camera has stabilized in an environment with a controlled and constant climate, the values of $T_{e}$ and $T_{d}$ and probably any other remaining $t$ dependent parameters, can be considered as constant so that the dependency of $s_{\text {tot }}$ on the variables $T$ and $t$ can be explicitly written as

$$
s_{\text {tot }}(T, t)=\frac{d s(T)}{d t} t+\frac{d}{d t}\left[s_{e}\left(T_{e}\right)+s_{d}\left(T_{d}\right)+\cdots\right] t+\Delta s .
$$

The blackbody contribution is separated from the rest of $t$ dependent terms in the above equation. The first goal is to determine the value of $s(T)$, when having only available the values of $s_{\text {tot }}(T, t)$ for given blackbody temperatures and integration times. This goal is addressed by removal of the blackbody during a measurement so that the remainder of the terms, resulting from the instrument being a real instrument as opposed to an ideal instrument, can be determined. With the values of these terms known, the value of $s(T)$ can easily be determined.

If a blackbody, or any other object, with temperature of $0 \mathrm{~K}$ is measured by an ideal instrument under a nonintervening environment, the instrument should have a zero output signal. When measured with a real instrument, like the CUI, the contribution of the first term in Eq. (23) should be zero, so that the measurement equation for this measurement now becomes

$$
s_{\text {tot }}(T=0 K, t)=\frac{d}{d t}\left[s_{e}\left(T_{e}\right)+s_{d}\left(T_{d}\right)+\cdots\right] t+\Delta s .
$$

After substitution of Eq. (24) into Eq. (23), the measurement equation becomes

$$
s_{t o t}(T, t)=\frac{d s(T)}{d t} t+s_{t o t}(T=0 K, t),
$$

and the value of $s(T)$ is obtained as

$$
\frac{d s(T)}{d t} t=s_{t o t}(T, t)-s_{t o t}(T=0 K, t),
$$

i.e. for a given integration time $t=t_{k}$,

$$
s\left(T, t_{k}\right)=s_{t o t}\left(T, t_{k}\right)-s_{t o t}\left(T=0 K, t_{k}\right) .
$$


Resolvement of the normalized spectral response $r(\lambda)$ can now be attemped by replacement of the signal from an ideal instrument $s(T)$ in Eq. (4) by $s\left(T, t_{k}\right)$, i.e.

$$
C_{L 2 s} \int_{\lambda_{a}}^{\lambda_{b}} r(\lambda) L_{b b}(T, \lambda) d \lambda=s_{t o t}\left(T, t_{k}\right)-s_{t o t}\left(T=0 K, t_{k}\right)
$$

The components of the signal vector $\boldsymbol{s}$ in Eq. (16) are, for the specific $t_{k}$ used during measurements, now set to

$$
s_{i}=s_{\text {tot }}\left(T_{i}, t_{k}\right)-s_{t o t}\left(T_{i}=0 K, t_{k}\right)
$$

after which $\boldsymbol{r}_{\alpha}$ can be resolved.

\subsubsection{Measurement setup and results}

Measurements were obtained after alignment of the CUI with each of two types of blackbodies, a cavity blackbody and a flat-plate calibrator, respectively. The $25 \mathrm{~mm}$ aperture cavity blackbody, an EOI model CS1250-100, has an emissivity of 0.97 to 0.99 with a stability of $\pm 0.25^{\circ} \mathrm{C}$ [28] and was calibrated by an accredited service provider over the temperature range of $50^{\circ} \mathrm{C}$ to $1200^{\circ} \mathrm{C}$ with a measurement uncertainty of $\pm 5{ }^{\circ} \mathrm{C}$. The Fluke 9133 portable infrared calibrator, shown in Fig. 9, has a target circular area with diameter of $57 \mathrm{~mm}$, stability of $\pm 0.1{ }^{\circ} \mathrm{C}$ and is capable of covering a temperature range of $-30{ }^{\circ} \mathrm{C}$ to $150{ }^{\circ} \mathrm{C}$. Its target area plate was painted with Nextel 811-21, having an approximate constant spectral emissivity of 0.97 [29] over the MWIR band. The accuracy of its temperature settings is not known.

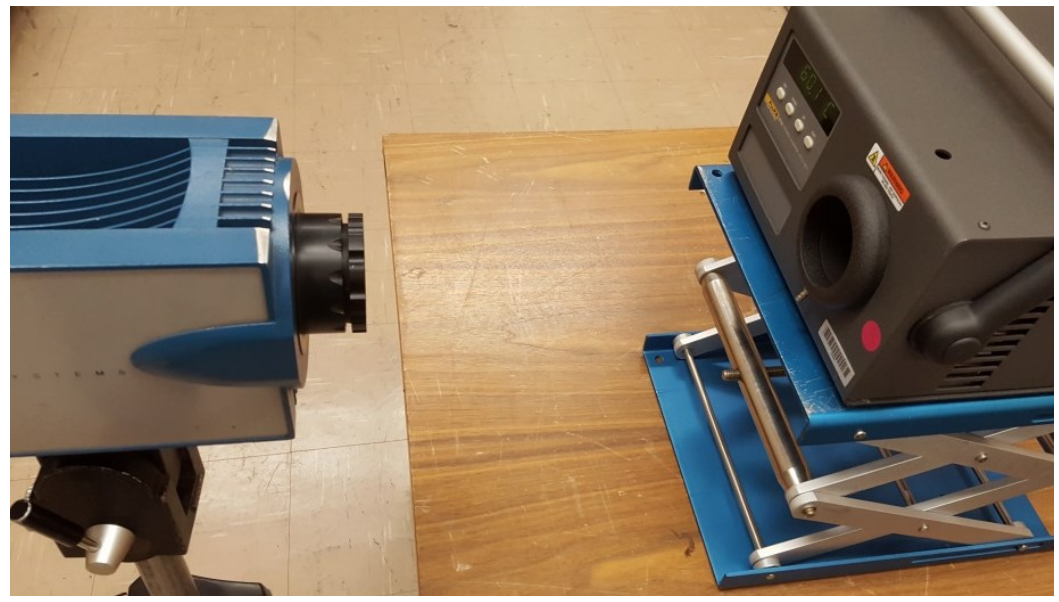

Fig. 9. The MWIR camera under investigation aligned with a flat-plate blackbody.

The output signal $s$ of the CUI is a 14-bit digitized voltage signal, with dynamic range of $0-16383\left(2^{14}\right.$ 1) digital levels $[D L]$, also called grey levels. Signal values above $14000 D L$ are not used in order to stay well clear from any saturation effects close to the camera's saturation level of $16383 \mathrm{DL}$. Before starting any measurements at a specific integration time $t$, a two-point non-uniformity correction (NUC) of the image pixels is done by means of measurements of a uniform flat-plate filling the FOV of the camera.

The cavity blackbody was used for high temperature measurements (above the upper limit of the flat-plate blackbody) using a short integration time, while the flat-plate blackbody was used for lower temperatures recorded with longer integration times. Measurements were done within a climate controlled environment after the CUI's internal temperature has stabilized, thereby attempting to keep the output signal originating from the camera's internal emission constant. The temperature of the camera detector is always kept constant during operation at $75 \mathrm{~K}$ by its own internal control system, thereby maintaining the dark current at a constant level.

To realize Equation (24), measurements of liquid nitrogen $(\sim 77 \mathrm{~K})$ over a short range of about $10 \mathrm{~cm}$ were considered as sufficient in order to ensure that no measurable, external blackbody/object radiation is observed by the camera. It was then attempted to do measurements of the blackbody over the widest temperature range possible (without the addition of a neutral density filter to the CUI) - for this reason three different integration times, collectively covering the lowest up to the highest temperatures measurable by the CUI, were used. The 
recording of a sequence of at least 100 image frames over 5 - 10 seconds of each of the blackbodies at each of the temperature settings $T_{i}$ followed. The values of $\Delta T$ was $\sim 5^{\circ} \mathrm{C}$ over the temperature range $\left[144,288{ }^{\circ} \mathrm{C}\right.$ for the integration time $t=30 \mu \mathrm{s}$ with $m=26, \sim 4{ }^{\circ} \mathrm{C}$ over the temperature range $[-22,144]{ }^{\circ} \mathrm{C}$ for $t=240 \mu \mathrm{s}$ (except for the highest temperatures for which $\Delta T$ was decreased to $\sim 2{ }^{\circ} \mathrm{C}$ ) with $m=45$ and $\sim 4{ }^{\circ} \mathrm{C}$ over the temperature range $[-22,78]{ }^{\circ} \mathrm{C}$ for $t=1000 \mu \mathrm{s}$ with $m=30$. In consideration of the uncertainties related to the blackbodies' settings, stability and exact output spectra, values of $\Delta T$ much smaller than $\sim 5^{\circ} \mathrm{C}$ was not considered sensible. Fig. 10 shows the measured output signal $s$ of a group of $4 \times 4$ pixels (averaged over the 16 pixels in each image frame as well as over the sequence of the recorded frames) near the centre of the recorded images as function of the blackbody temperature $T$ at the different CUI integration times respectively. It can be seen that the three integration times span different blackbody temperature ranges and that the number of blackbody temperatures at which measurements were made within these ranges differ.

The internal temperature of the camera was kept as constant as possible, although during the $t=30 \mu \mathrm{s}$ measurements, the camera temperature still varied by up to $2{ }^{\circ} \mathrm{C}$; during the $t=240 \mu \mathrm{s}$ and $1000 \mu \mathrm{s}$ measurements, it was possible to maintain the camera temperature variation below $0.3^{\circ} \mathrm{C}$. Although an NUC was applied for each integration time, such a correction is rarely ever perfect over a wide range of recorded temperatures, so that small variations between pixel values will persist. In spite of an imperfect NUC, different selections of pixels did not influence the results significantly - experimentation with $D L$ values obtained from single pixels or groups of pixels at different positions in the image did not have any significant influence on the solutions to be presented in Section 3.2.3. However, it is expected that as the quality of the NUC worsens, the measurement results will be negatively influenced with a subsequent negative effect on the spectral response resolutions.

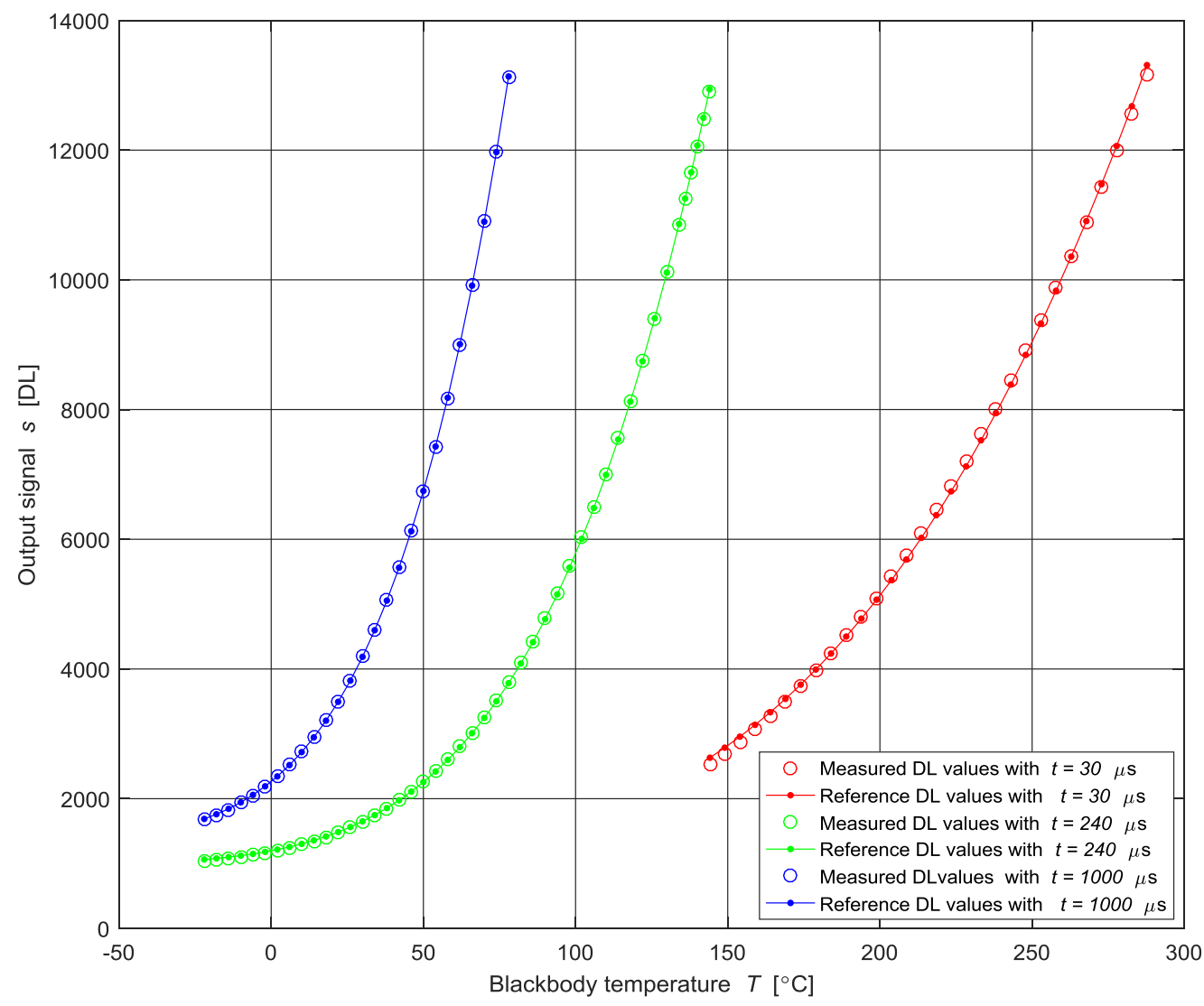

Fig. 10. The measured instrument output signal $s$ (circular markers) as a function of blackbody temperature. The cavity blackbody was used for integration time $t=30 \mu \mathrm{s}$, while the flat plate blackbody was used for $t=240 \mu \mathrm{s}$ and $t=$ $1000 \mu \mathrm{s}$. Also shown is the reference $s$ values (solid markers and lines) obtained by fitting the measured $s$ to the calculated $L_{p n}$ (shown in Fig. 11) by using the reference spectrum $r_{i n s t r 1}$ in Section 3.1. Note the small differences between the measured and reference $s$ values, especially for $t=30 \mu \mathrm{s}$. 
Fig. $11 \mathrm{a}, \mathrm{c}$ and e show the measured signals $s$ at the three different values of $t v s$. the peak-normalized values $L_{p n}$, with $L_{p n}$ calculated from Eq. (6) by employing the reference response spectrum $r_{\text {instr } 1}$ (of Section 3.1). As mentioned in Section 3.1, $r_{\text {instr } 1}$ is considered as the most accurate representation of the actual response spectra of the CUI, and a linear relationship is expected between $s$ and $L_{p n}$, especially for this CUI [26]. Deviations from such a linear relationship are therefore considered to be the result of measurement inaccuracies resulting from the blackbody source and CUI and not of an inadequate/inaccurate responsivity spectrum of the CUI (as might be the case as stated at the end of Section 2.3).

A least squares straight line fit was applied to the measured $s$ and $L_{p n}$ data in order to determine the deviation of the measured $s$ values from the fitted line. The difference between the measured values and the values predicted by the fitted line, which will be referred to as the reference $s$ values, is shown in Fig. $11 \mathrm{~b}$, $\mathrm{d}$ and $\mathrm{f}$ for the three respective integration times, with the associated Root Mean Square Error (RMSE) also indicated in the figure caption. It is expected that more accurately known spectra from the blackbody instrument and a more stable measuring instrument would result in an improved $s v s$. $L_{p n}$ straight line fit with a smaller RMSE. 
(a)

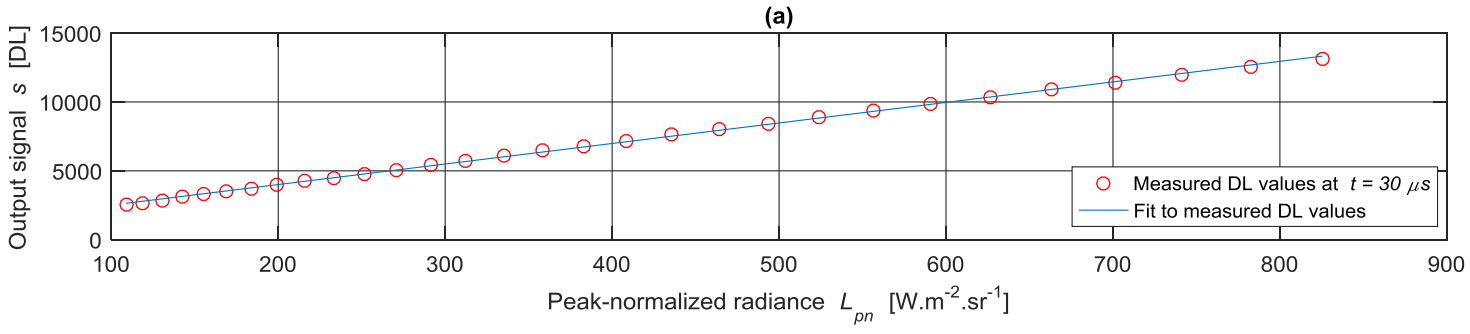

(b)

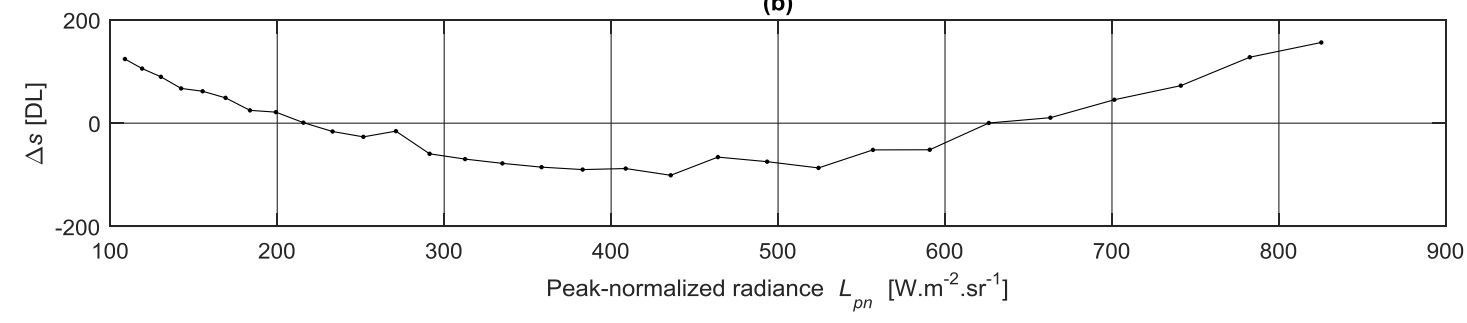

(c)

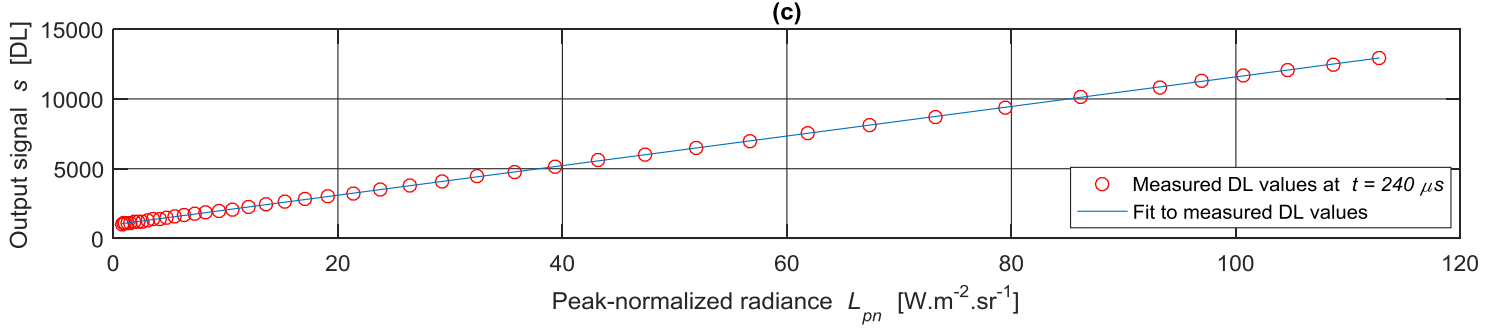

(d)

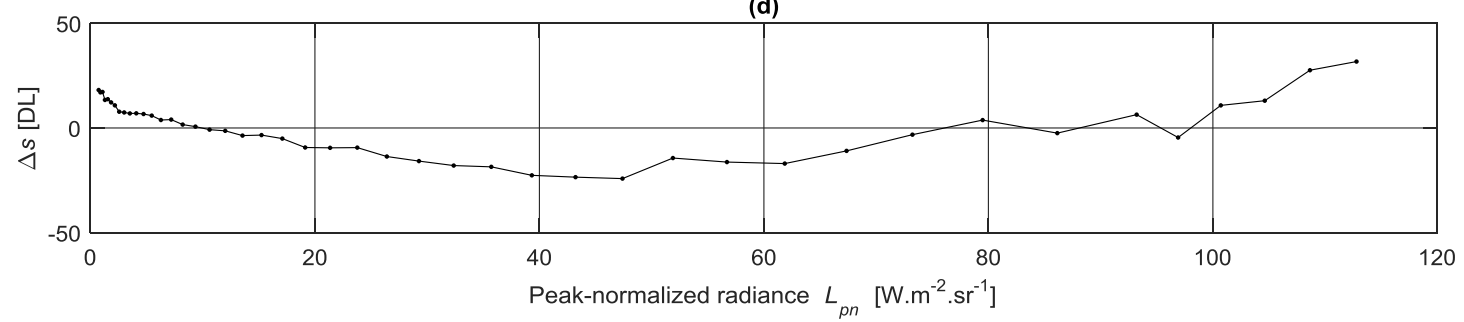

(e)

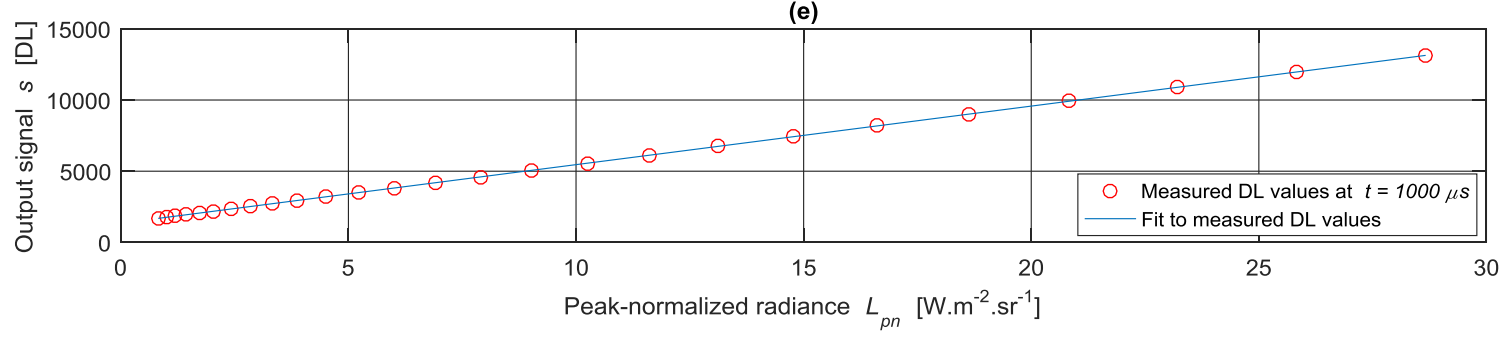

(f)

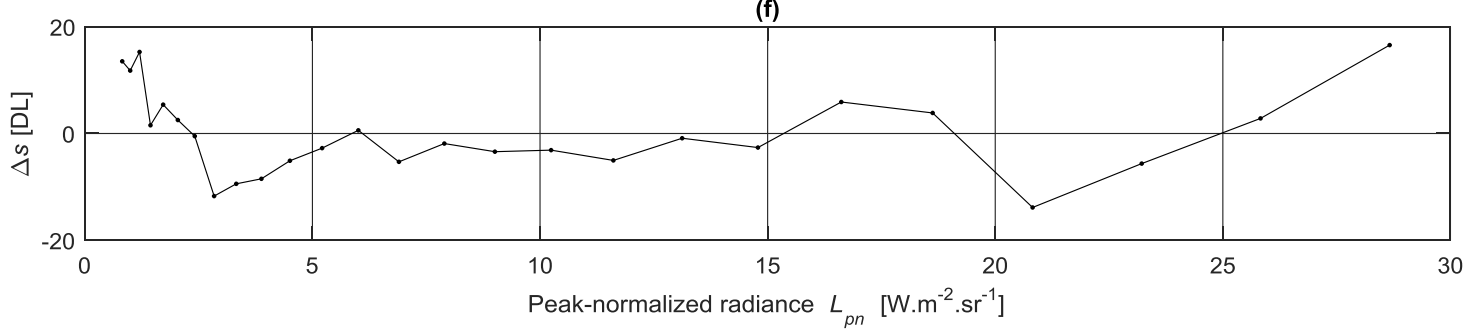

Fig. 11. The camera measured output signal $s$ (red circles) $v s . L_{p n}(T)$, with $L_{p n}(T)$ calculated from Eq. (6) using $r_{i n s t r 1}$ for (a) $t=30 \mu \mathrm{s}$, (c) $t=240 \mu \mathrm{s}$ and (e) $t=1000 \mu \mathrm{s}$. The straight blue line in each graph is the least squares fit to the data. The difference between the straight line fit and the measured signal values for the three different $t$ values used in (a), (c) and (e) are shown in (b) with $R M S E=74.4$ (0.45\% of dynamic range), (d) with $R M S E=13.3(0.08 \%$ of dynamic range) and (f) with $R M S E=7.8(0.05 \%$ of dynamic range) respectively. 


\subsubsection{Resolving the camera normalized spectral response}

An important difference between the modelled measurements of Section 2.2 and the real measurements obtained with the CUI in this section is the measurement inaccuracies present in the real recordings. As mentioned in Section 2.1 when Wing [15] was quoted, small changes in the measured data may produce wild behaviour in the solution so that the solution have to be tamed using various means. Although great care was taken doing the CUI calibration type measurements as accurately as possible with the available equipment, non-ideal features of the measurements existed in the form of uncertainties of the emissivity, stability and temperature settings of the two blackbodies. Furthermore, variations occurred in the internal temperature of the CUI during the measurements (which would have an influence on the recorded DL values), in spite of a conscience effort to control and stabilize its environment. However, processing of the different sets of data obtained for the different integration times from the two different types of blackbodies used, with all the uncertainties involved, is considered to be a good test for the consistency of the proposed technique.

Recovery of the CUI response spectrum was attempted by using the measured DL values as shown in Fig. 10, as well as the reference $\mathrm{s}$ (DL) values obtained from the straight line fit to the data which can be considered as a more accurate set of measurements. Recovered spectra were obtained for a range of $\alpha$ values for each integration time, using as modelling parameter values both a wide wavelength range covering $1-12 \mu \mathrm{m}$ and a narrower range covering 3-6 $\mu \mathrm{m}$ during the application of Eq. (16). The results for the reference DL values are discussed first, where after the solutions for the measured DL values are discussed.

Starting with the CUI reference DL recordings done with integration time $t=30 \mu \mathrm{s}$ of $m=26$ temperatures over the range $[144,288]{ }^{\circ} \mathrm{C}$ with $\Delta T \approx 5^{\circ} \mathrm{C}$, solutions of the spectral response were obtained with the modelling parameters $n=26$ (i.e. $\Delta \lambda \approx 0.38 \mu m$ ) and $\left[\lambda_{1}, \lambda_{m}\right]=1-12 \mu m$ for $\alpha=\left\{10^{1}, 10^{0.5}, 10^{0}, \ldots, 10^{-26}\right\}$. The logarithmic values of the solution norms $\left\|C_{L 2 s} \boldsymbol{r}\right\|$ and residual norms $\left\|C_{L 2 s} \boldsymbol{L r}-\boldsymbol{s}\right\|$, as associated with the solutions resulting from each $\alpha$ value, were plotted against each other to obtain the L-curves as shown in Fig. 12a. The corner position obtained form $\alpha=10^{0}=1$ was easily identified and are marked in the figure. The recovered response spectrum associated with this $\alpha$ value is shown by the black curve in Fig. 13a. Identification of the active response region within the wide band covering $1-12 \mu \mathrm{m}$ can easily identified as the $3-6 \mu \mathrm{m}$ MWIR band, so that the modelling wavelength range was subsequently narrowed down to $3-6 \mu \mathrm{m}$. The L-curve for this narrowed wavelength band, with a corner point associated with $\alpha=10^{-2}$, is shown in Fig. 12b with the resulting recovered spectrum shown by the black curve in Fig. 13b. It can be seen that this recovered spectrum, which represents the best solution for all the $\alpha$ values, is a reasonable representation of the known $r_{\text {instr } 1}$ spectrum shown in blue.

The recovered spectra associated with the corner point $\alpha$ values in the remaining figures Fig. $12 \mathrm{c}$ to Fig. $12 \mathrm{f}$ for the reference DL recordings with integration times $t=240 \mu \mathrm{s}$ and $1000 \mu \mathrm{s}$, as solved over $\left[\lambda_{1}, \lambda_{m}\right]=$ $1-12 \mu \mathrm{m}$ and $3-6 \mu \mathrm{m}$, are shown in Fig. 12c to Fig. 12f respectively. The similarities in the recovered response spectra solved over the $1-12 \mu \mathrm{m}$ range are easily observed, as is the case for the spectra solved over $3-6 \mu \mathrm{m}$.

The spectra were then also recovered for the measured DL values, i.e. from the 'less accurate' unadjusted measurements. In the same fashion as described above, L-curves were obtained from solutions associated with different $\alpha$ values - these L-curves are shown in Fig. 14. The solutions obtained for the $\alpha$ values of the corner points of the curves are plotted in red in Fig. 13. It was found that not all spectra recovered using these $\alpha$ values gave acceptable results - this was the case for the spectra shown in Fig. 13d and Fig. 13f. Acceptable solutions were found by visual inspection for the data points indicated in green on the L-curves; the solutions calculated with the associated $\alpha$ values are also plotted in green in Fig. 13d and Fig. 13f. Also shown in Fig. $13 \mathrm{e}$ is the slightly improved solution of the response spectrum when the $\alpha$ value of the data point next to the corner point of the L-curve in Fig. 14e was used. 
(a)

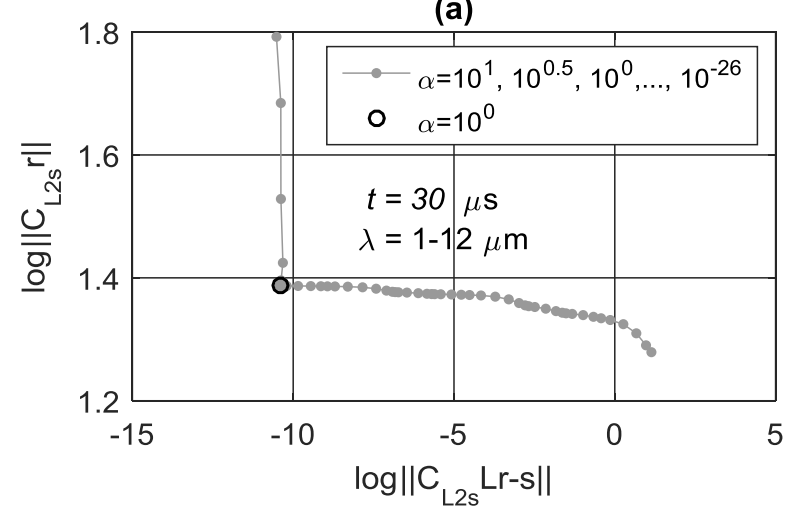

(c)

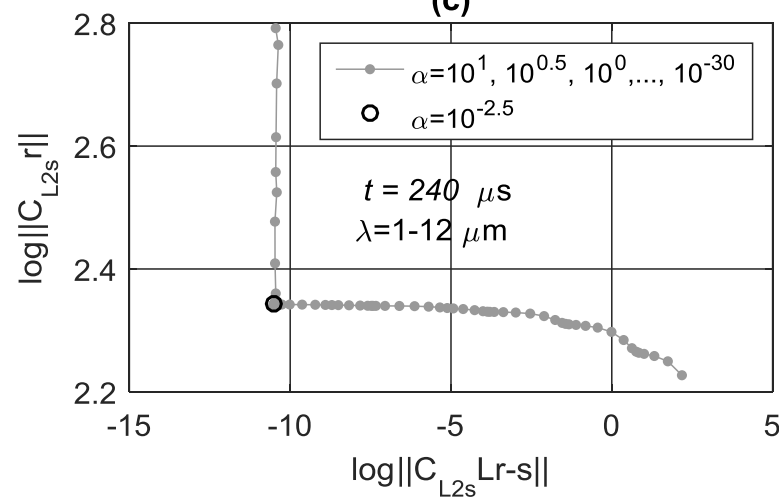

(e)

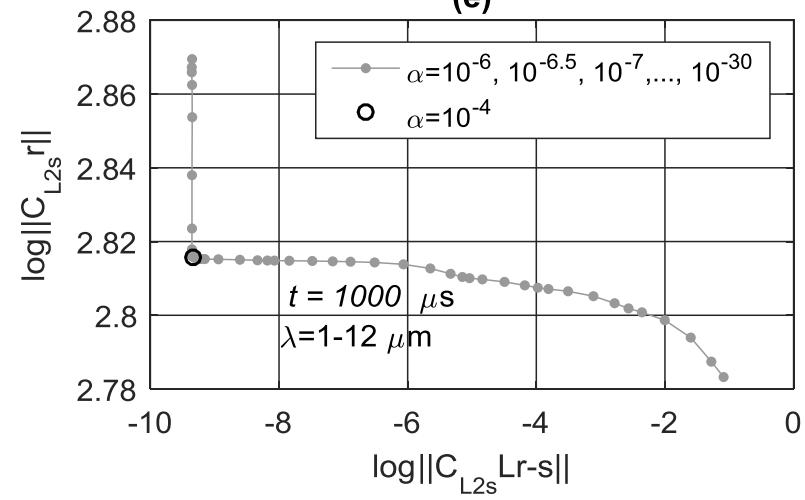

(b)

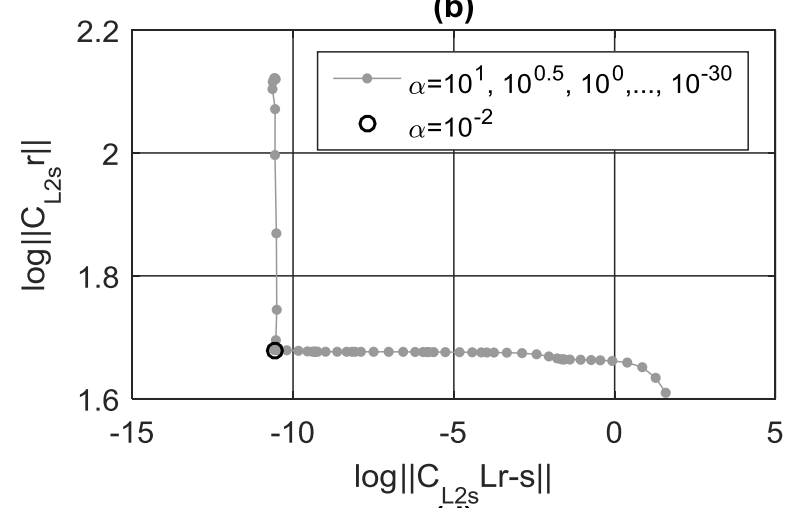

(d)
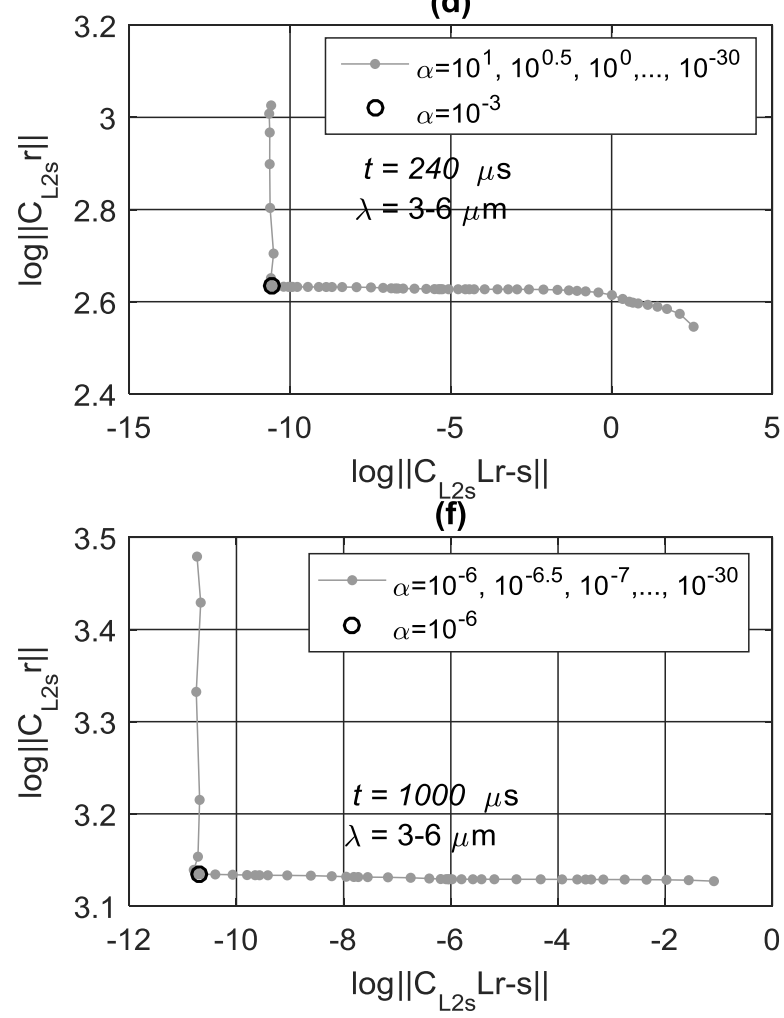

Fig. 12. The L-curves obtained from the response spectra as recovered from the reference data. The integration time and wavelength region used during recovery are shown for each graph. The $\alpha$ values used for the creation of the L-curves are also indicated (grey solid markers), as well as the $\alpha$ value associated with the corner point of the curve (black circle). 
(a)

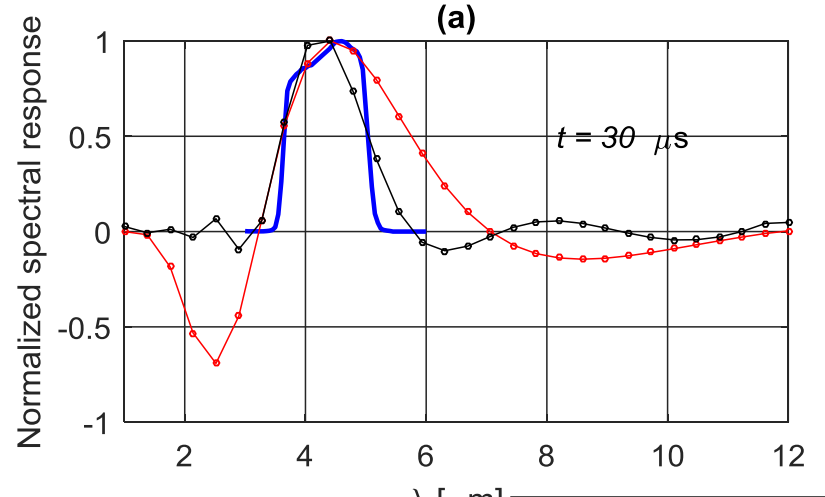

$\lambda[\mu \mathrm{m}] \longrightarrow$ Reference response $r_{\text {instr1 }}$

(c)

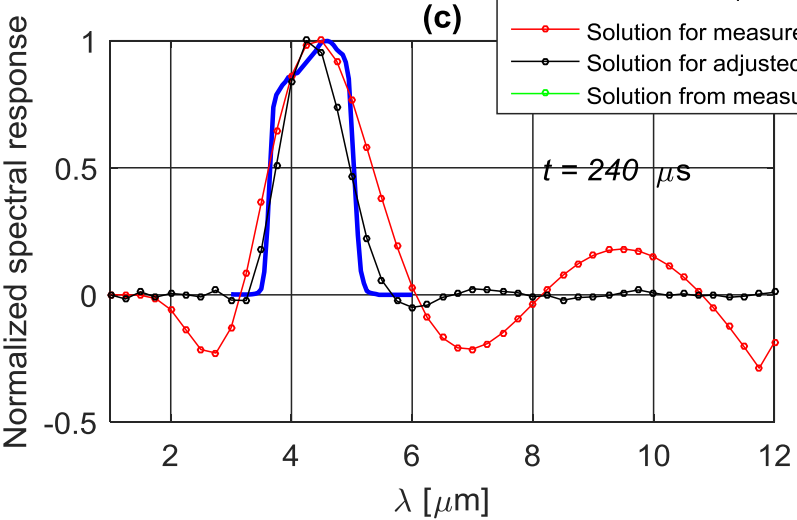

(e)

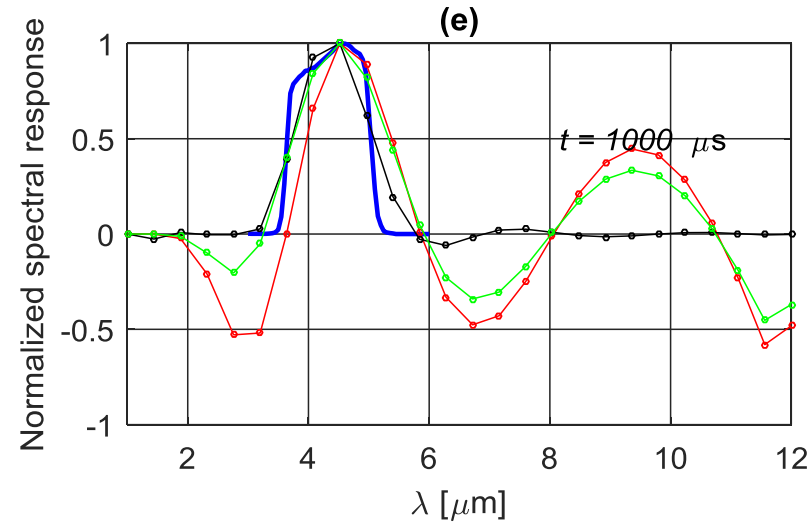

(b)

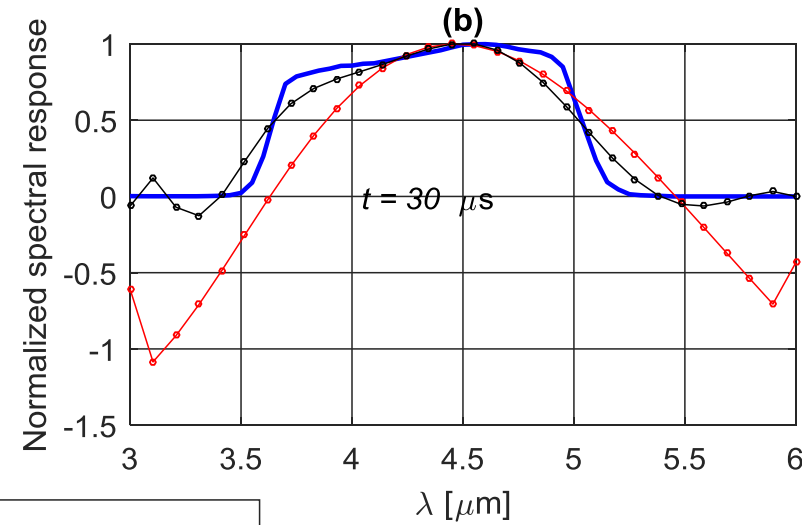

(d)
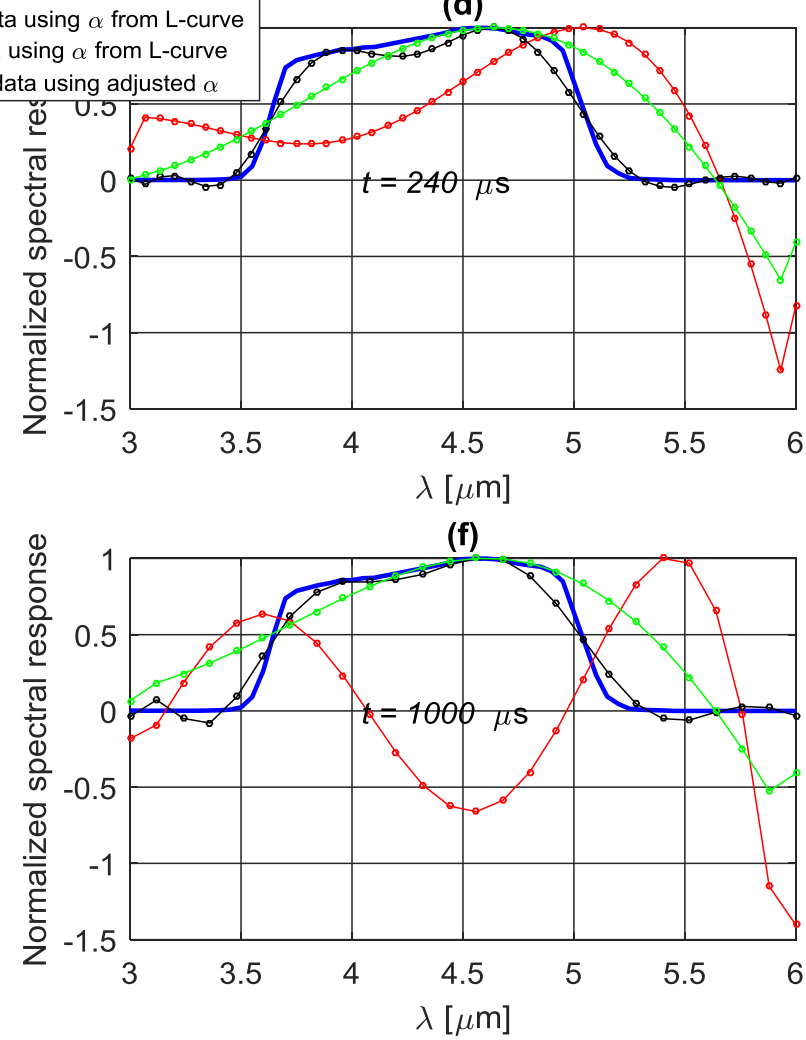

Fig. 13. The known spectral response $r_{i n s t r 1}$ (in blue) and the solutions to the spectral response of the CUI from measurements (in red) and theoretically adjusted reference measurements (in black) at different integration times, as indicated on the plots, and for the different wavelength ranges of $\lambda=1-12 \mu \mathrm{m}$ and $\lambda=3-6 \mu \mathrm{m}$ used as input. The applicable parameters used in obtaining these solutions are shown in the corresponding sub-figures of Fig. 12 and Fig. 14. 
(a)

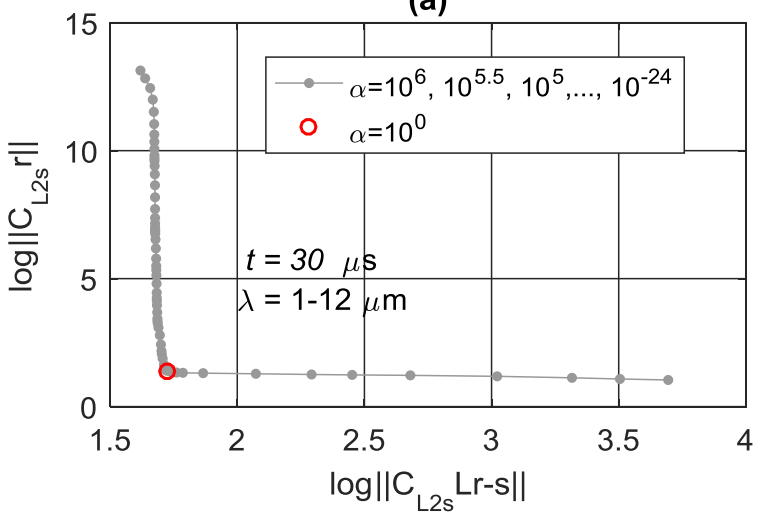

(c)

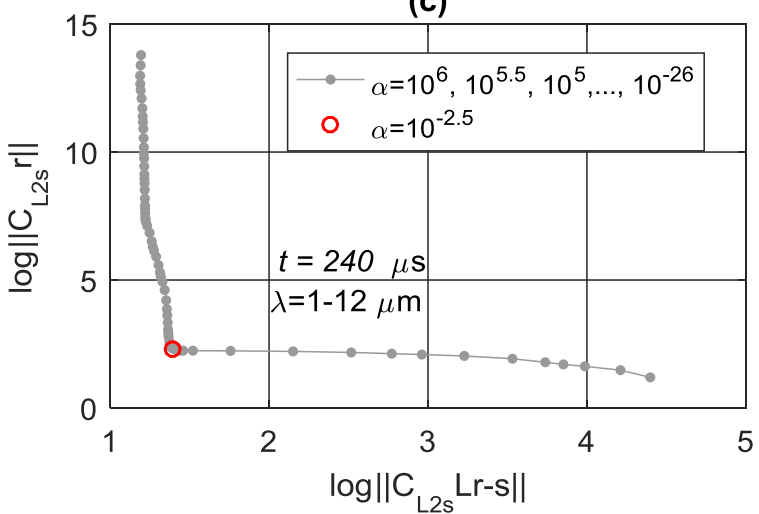

(e)

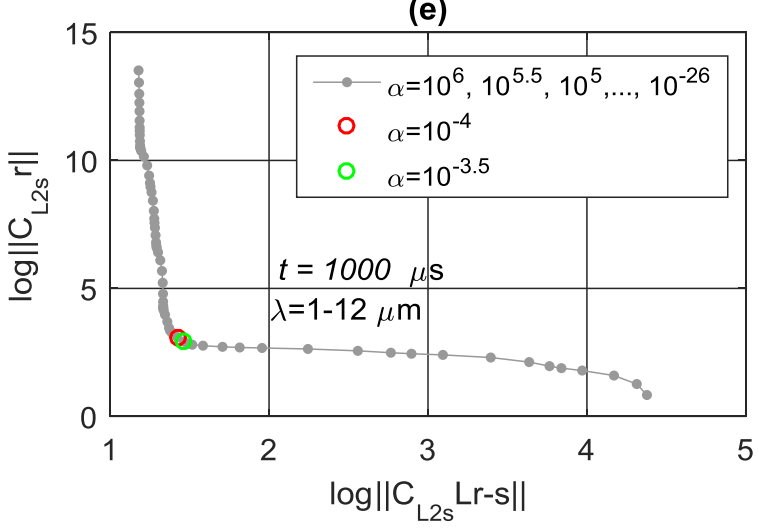

(b)

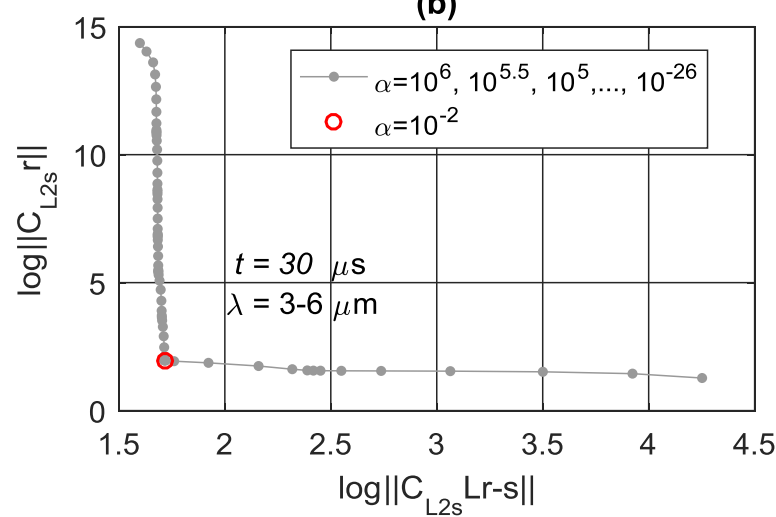

(d)

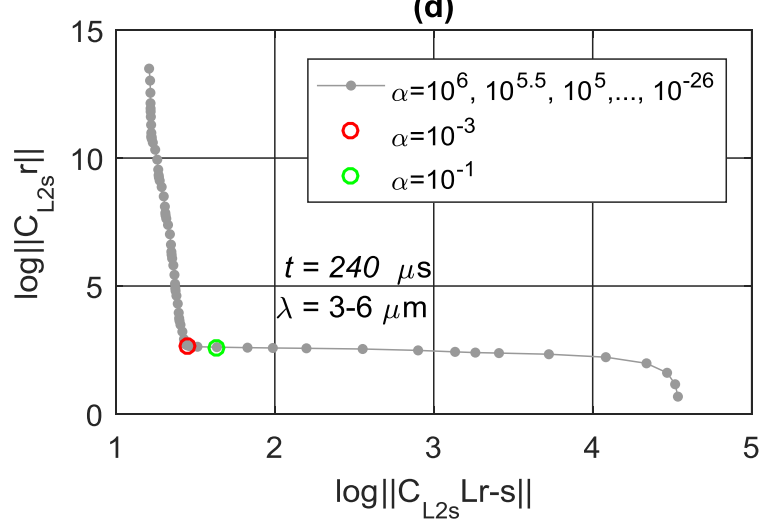

(f)

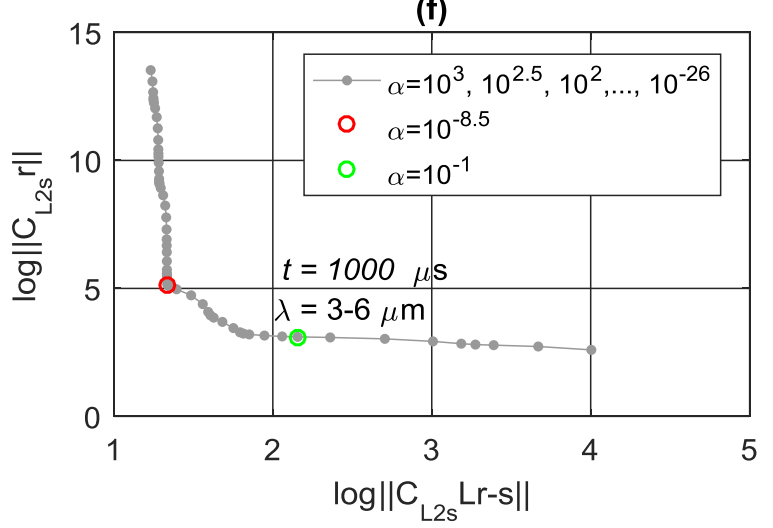

Fig. 14. The L-curves obtained from the response spectra as recovered from the measured data. The integration time and wavelength region used during recovery are shown for each graph. The $\alpha$ values used for the creation of the L-curves are also indicated (grey solid markers), as well as the $\alpha$ value associated with the corner point of the curve (red circle) and the $\alpha$ value resulting in the best visual agreement with the known response spectrum (green circle).

In Fig. 15 the theoretical and experimentally CVF determined (reference) response spectra of Section 3.1 are shown together with some of the resolved spectra based on the solutions shown in Fig. 13. Finalization in resolving the spectra were done according to procedure described in Section 2.3, i.e. all negative values were changed to zero and any non-zero responses separated from the main response settled in the $3.5-5.5 \mu \mathrm{m}$ region were also changed to zero. A few remarks can be made regarding the shown spectra:

- There is a reasonable (though not perfect) agreement in the wavelength position of maximum values of the resolved spectra with that of the reference spectrum $r_{\text {instr } 1}$.

- The theoretically adjusted signal values compare relatively well with $r_{i n s t r 1}$, and compare better than the theoretically calculated response spectrum $r_{i n s t r}$.

- In comparing the resolved spectrum using the unadjusted signal measurements with that obtained by using the theoretically adjusted signal measurements, it can inferred that measurements made with improved accuracy would result in improved spectral response recovery. 


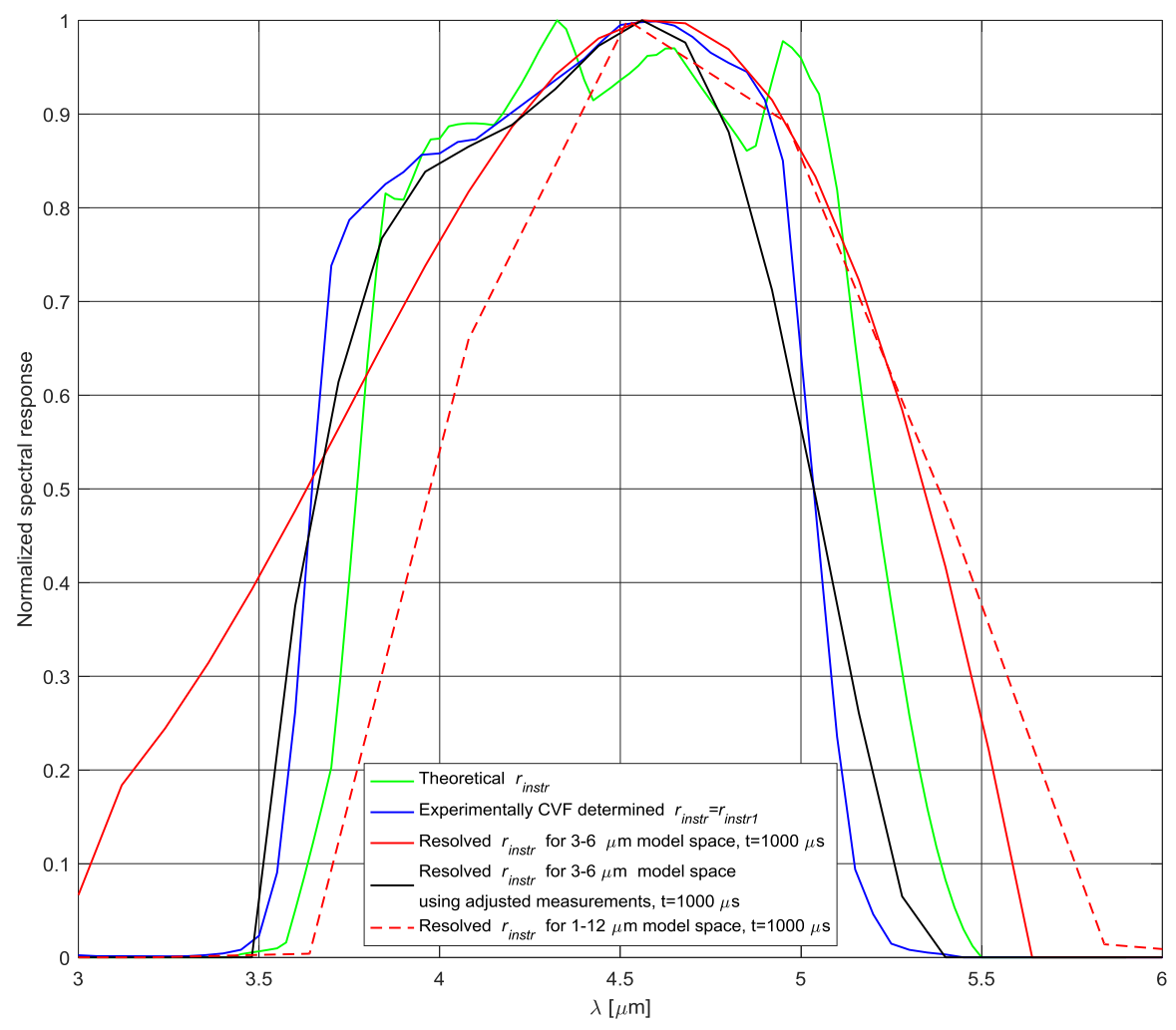

Fig. 15. The resolved spectral response for the CUI together with the theoretical and experimentally determined responses as presented in Section 3.1.

\section{CONCLUSION}

A technique was presented for resolving the spectral response of a wide-band infrared measuring instrument based on calibration type measurements of a blackbody at different temperatures and the mathematical technique as outlined in Section 2. The technique addresses the solution of a Fredholm integral equation of the first kind, which describes blackbody measurements by the measuring instrument. It was shown that during the attempt to solve the instrument response spectrum, subject matter knowledge is required in tailoring the inputs to the technique as well as the initial solution produced by the technique, and hence this process is referred to as 'resolving' the response spectrum.

A significant part of the mathematical technique is centred on the Tikhonov regularization method used during the inversion of the radiance matrix in the Fredholm equation, which is constructed from modelled values of blackbody spectra. The suitability of the blackbody spectral model, and thus the accuracy of the radiance matrix, is subject to the fidelity of the commercially available blackbody source instruments in accurately representing Planck's law in their emission spectra at different temperature settings.

The technique was first tested by means of error-free, synthetically generated instrument output signals resulting from a 'measured' blackbody source. It was shown that the solutions obtained from the synthetic signals for the response spectra were in reasonable agreement with the response spectra used in the generation of these signals. The accuracy of the resolved spectra is highly dependent on the value of the regularization parameter $\alpha$-a larger value of $\alpha$ results in a more stable solution, but with lower accuracy, whereas a smaller value of $\alpha$ results in a more accurate solution, but with an increasing tendency of instability. The method of visual inspection as well as the L-curve method were explained and used to obtain optimal $\alpha$ values, which can typically cover a range of values.

It is stated by Wing [15] that the optimal value of $\alpha$ is not only determined by the matrix to be inverted ( $\tilde{\boldsymbol{L}}$ in this work), but also by the statistical distribution of the error in the data and the data itself; this can be confirmed by the attempt to recover the response spectrum of an IR camera by using real calibration type measurements, considered to contain relative large uncertainties, that were done with the camera. In this respect, not all response spectra could be successfully recovered by the L-curve method, and the method of 
visual inspection had to be employed in order to obtain reasonable response spectra. The measurements made were then also adjusted to be representative of more accurate measurements, after which the instrument spectrum could be recovered with great success by using only the L-curve method. In consideration of these results, together with the results of the error free synthetic data, it seems that the technique requires a high degree of accuracy in the measurements in order to be applied successfully. Quantification of the required degree of accuracy, as well as the investigation into possible improvements of the presented method, is foreseen in future research.

In the use of the response spectrum during normal data reduction activities for obtaining the IR characteristics of objects of interest (as described in Section 1), differences in the actual response spectrum and the resolved response spectrum might, under certain operational circumstances, become less critical - for example, for measurements of objects over long distances, the atmosphere introduces a cut-off for radiation with $\lambda>$ $5 \mu \mathrm{m}$ in the MWIR band (easily verifiable using radiative transfer code like Modtran ${ }^{\mathrm{TM}}$ ). Such an environmental filter would make the over-extension of a resolved response spectrum to wavelengths longer than $5 \mu \mathrm{m}$ (see e.g Fig. 15) of little consequence during data reduction when used together with the modelled atmospheric spectral transmission.

Other infrared sources with known emission spectra can also be used after appropriate modification of the radiance matrix, which could result in a more stable matrix inversion. Also, other methods than Tikhonov regularization for stable matrix inversion exist and need to be investigated for possible improved solutions when using measurements characterised by non-negligible margins of error.

Application of the technique can, besides instrument spectral response recovery, also be extended to optical filter transmission characterization if high accuracy measurements are possible, or at least filter transmission band identification of unspecified filters when only lower accuracy measurements are possible.

\section{ACKNOWLEDGEMENTS}

The author would like to acknowledge and thank Dr. WH Gunter of the Institute of Maritime Technology in Simon's Town, South Africa, for supplying data on the experimentally determined response spectra of the camera under discussion. Special thanks are also extended to my colleagues Hannie van den Bergh and Neels Brink, as well as to Prof. A Kijko of the University of Pretoria, for valuable discussions regarding the topic of this research. This research did not receive any specific grant from funding agencies in the public, commercial, or non-profit sectors.

\section{REFERENCES}

[1] J.M. Palmer, B.G. Grant, Chapter 1 - Introduction to radiometry, in: Art Radiom., SPIE Press, Bellingham, 2009: pp. 1-9.

[2] C.J. Willers, Chapter 3 - Sources, in: Electro-Optical Syst. Anal. Des. A Radiom. Perspect., SPIE The International Society for Optical Engineering, Bellingham, 2013: pp. 57-96.

[3] A. Rogalski, K. Chrzanowski, Infrared devices and techniques (revision), Opto-Electronics Rev. 10 (2002) 111-136.

[4] A. Rogalski, Progress in focal plane array technologies, Prog. Quantum Electron. 36 (2012) 342-473. doi:10.1016/j.pquantelec.2012.07.001.

[5] W.L. Wolfe, Chapter 14 - Radiometric temperatures, in: Introd. to Radiom., SPIE, 1000 20th Street, Bellingham, WA 98227-0010 USA, 1998: pp. 143-149. http://ebooks.spiedigitallibrary.org/book.aspx?doi=10.1117/3.287476.

[6] S.J.P. Retief, P. Smit, M.M. Dreyer, Mid-wave infrared characterization of an aircraft plume, Saudi Int. Electron. Commun. Photonics Conf. 2011, SIECPC 2011. (2011) 1-6. doi:10.1109/SIECPC.2011.5876932.

[7] J.M. Palmer, B.G. Grant, Chapter 5 - Detectors of Optical Radiation, in: Art Radiom., SPIE Press, Bellingham, 2009: pp. 127-213.

[8] V.B. Podobedov, G.P. Eppeldauer, L.M. Hanssen, T.C. Larason, Calibration of spectral responsivity of IR detectors in the range from $0.6 \mu \mathrm{m}$ to $24 \mu \mathrm{m}$, in: B.F. Andresen, G.F. Fulop, C.M. Hansen, P.R. Norton (Eds.), Infrared Technol. Appl. XLII, 2016: p. 98190P1-98190P11. 
[9] J.M. Palmer, B.G. Grant, Chapter 7 - Radiometric measurement and calibration, in: Art Radiom., SPIE Press, Bellingham, 2009: pp. 241-268.

[10] C.J. Willers, Chapter 5 - Optical Detectors, in: Electro-Optical Syst. Anal. Des. A Radiom. Perspect., SPIE - The International Society for Optical Engineering, Bellingham, 2013: pp. 63-86. doi:10.1002/9781118014103.ch4.

[11] W.L. Wolfe, Chapter 9 - Normalization, in: Introd. to Radiom., SPIE, 1000 20th Street, Bellingham, WA 98227-0010 USA, 1998. http://ebooks.spiedigitallibrary.org/book.aspx?doi=10.1117/3.287476.

[12] C.L. Wyatt, Radiometrie Calibration : Theory and Methods, (1978).

[13] F.E. Nicodemus, Normalization in Radiometry, Appl. Opt. 12 (1973) 2960-2973.

[14] C.J. Willers, Chapter 7 - Radiometry Techniques, in: Electro-Optical Syst. Anal. Des. A Radiom. Perspect., SPIE - The International Society for Optical Engineering, Bellingham, 2013.

[15] G.M. Wing, A Primer on Integral Equations of the First Kind, Society for Industrial and Applied Mathematics, 1991. doi:10.1137/1.9781611971675.

[16] W. Press, B. Flannery, S. Teukolsky, W. Vetterling, Numerical Recipes in C: The Art of Scientific Computing, 2nd ed., Cambridge University Press, 2002.

[17] R.C. Aster, B. Borchers, C.H. Thurber, Parameter Estimation and Inverse Problems, 2nd ed., Elsevier, 2013. doi:10.1016/B978-0-12-385048-5.00002-1.

[18] P. Yang, X.-W. Feng, W.-J. Liang, K.-S. Wu, Numerical Solutions of Inverse Black Body Radiation Problems with Gaussian-Laguerre Quadrature Formula, Int. J. Theor. Phys. 54 (2015) 519-525.

[19] C.W. Groetsch, Integral equations of the first kind, inverse problems and regularization: a crash course, J. Phys. Conf. Ser. 73 (2007) 012001.

[20] S.J. Gibowicz, A. Kijko, Chapter 4 - Location of Seismic Events in Mines, in: An Introd. to Min. Seismol., Academic Press, Inc., 1994: pp. 48-71.

[21] M.S. Zhdanov, Chapter 2 - Ill-posed problems and the methods of their solution, in: Inverse Theory Appl. Geophys. 2nd Ed., Elsevier, 2015: pp. 33-61.

[22] P.C. Hansen, Chapter 7 - Parameter-Choice Methods, in: Rank. Discret. Ill-Posed Probl., Society for Industrial and Applied Mathematics, 1998: pp. 175-208.

[23] J.B. Shumaker, Linearity Considerations and Calibrations, Chapter 11, in: Self-Study Man. Opt. Radiat. Meas., National Buro of Standards (U.S.) Tech. Note 910-3, 1984: p. 31.

[24] C.L. Wyatt, Radiometric Calibration: Theory and Methods, Academic Press, Inc., 1978.

[25] W.H. Gunter, Institute for Maritime Technology, Personal communication, 2006.

[26] L. Shkedy, O. Amir, Z. Calahora, J. Oiknine-Schlesinger, I. Szafranek, Temperature dependence of spatial noise in InSb focal plane arrays, in: E.L. Dereniak, R.E. Sampson (Eds.), Infrared Dectectors Focal Pl. Arrays VI, SPIE - The International Society for Optical Engineering, 2000: pp. 481-488. doi:10.1117/12.391762.

[27] J.K. Ji, J.R. Yoon, K. Cho, Nonuniformity correction scheme for an infrared camera including the background effect due to camera temperature variation, Opt. Eng. 39 (2000) 936-940.

[28] Electro Optical Industries Inc., CS SERIES High Temperature Sources specification sheet, 1999.

[29] A. Adibekyan, E. Kononogova, C. Monte, J. Hollandt, High-Accuracy Emissivity Data on the Coatings Nextel 811-21, Herberts 1534, Aeroglaze Z306 and Acktar Fractal Black, Int. J. Thermophys. 38 (2017) 1-14.

[30] A. Neumaier, Solving Ill-Conditioned and Singular Linear Systems: A Tutorial on Regularization, SIAM Rev. 40 (1998) 636-666.

\section{Highlights}

- A technique for obtaining spectral responses of infrared instruments is presented.

- Monochromator type measurements are not required.

- Calculations demanding instrument spectral specifications are not required.

- Accurate calibration type measurements are required.

- A mathematical technique for processing the measurements is presented. 